\title{
An étalé space construction for stacks
}

\author{
DAVID CARCHEDI
}

\begin{abstract}
We generalize the notion of a sheaf of sets over a space to define the notion of a small stack of groupoids over an étale stack. We then provide a construction analogous to the étale space construction in this context, establishing an equivalence of $2-$ categories between small stacks over an étale stack and local homeomorphisms over it. These results hold for a wide variety of types of spaces, for example, topological spaces, locales, various types of manifolds, and schemes over a fixed base (where stacks are taken with respect to the Zariski topology). Along the way, we also prove that the 2-category of topoi is fully reflective in the 2-category of localic stacks.
\end{abstract}

22A22, 58H05, 53C08; 18B25, 14A20, 18F20

\section{Introduction}

The purpose of this article is to extend the theory of sheaves of sets over spaces to a theory of small stacks of groupoids over étale stacks. We provide a construction analogous to the étalé space construction in this context and establish an equivalence of 2-categories between small stacks over an étale stack and local homeomorphisms over it.

Étale stacks model quotients of spaces by certain local symmetries, and their points can posses intrinsic (discrete) automorphism groups. A more or less direct consequence of the existence of points with nontrivial automorphism groups is that étale stacks form not only a category, but a bicategory. A widely studied class of such stacks in the differentiable setting is orbifolds. Orbifolds arose initially out of foliation theory, but currently enjoy a wide variety of other uses. More generally, differentiable étale stacks are an important class of stacks as they include not only all orbifolds, but more generally, all stacky leaf spaces of foliated manifolds. The passage from spaces to étale stacks is a natural one as such a passage circumvents many obstructions to geometric problems. For example, it is not true that every foliation of a manifold $M$ arises from a submersion $f: M \rightarrow N$ of manifolds, however, it is true that every foliation on $M$ arises from a submersion $M \rightarrow \mathcal{X}$, where $\mathcal{X}$ is allowed to be an étale differentiable stack; see Moerdijk [17]. Similarly, it is not true that every Lie algebroid over a manifold $M$ integrates to a Lie groupoid $\mathcal{G} \rightrightarrows M$ (Crainic and Fernandes [4]) however 
it is true when the arrow space $\mathcal{G}$ is allowed to be an étale differentiable stack; see Tseng and Zhu [20]. Continuing this theme, it is not always possible for a stack over a space to be realized as the sheaf of sections of some local homeomorphism from another space (unless it is actually a sheaf of sets), but it is possible once this other space is allowed to be an étale stack, as we show in this paper. Étale stacks are also a natural setting to consider sheaves in, as the results of Pronk [19] imply that étale stacks are faithfully represented by their topos of sheaves.

Recall that for a topological space $X$, a sheaf over $X$ is a sheaf over its category of open subsets, $\mathcal{O}(X)$, where the arrows are inclusions. The corresponding topos is denoted as $\operatorname{Sh}(X)$. For small (pre-)sheaves over $X$, there is an étalé space construction: Given a presheaf $F$ over $X$, there exists a topological space $\underline{L}(F)$ and a local homeomorphism

$$
L(F): \underline{L}(F) \longrightarrow X
$$

such that for every open subset $U$ of $X$, sections of the map $L(F)$ over $U$ are in bijection with elements of $a F(U)$, where $a F$ is the sheaf associated to $F$. The space $\underline{L}(F)$ (together with its map down to $X$ ) is called the étalé space of $F$. More precisely there is a pair of adjoint functors

$$
\operatorname{Set}^{\mathcal{O}(X)^{o p}} \stackrel{\Gamma}{L} \mathbb{T O P} / X
$$

such that

$$
L(U)=U \hookrightarrow X .
$$

Here $L$ takes a presheaf to its étalé space and $\Gamma$ takes a space over $X$ to its sheaf of sections. This adjunction restricts to an equivalence

$$
\operatorname{Sh}(X) \underset{L}{\stackrel{\Gamma}{\leftrightarrows}} \operatorname{Et}(X)
$$

between the category of sheaves over $X$ and the category of local homeomorphisms over $X$.

Remark The étalé space construction works for a larger class of spaces than just topological spaces. For example, if $X$ were a smooth manifold, then the étale space of a sheaf $F$ would inherit the canonical structure of a (possibly non-Hausdorff) manifold, making the projection

$$
L F: \underline{L F} \rightarrow X
$$

a local diffeomorphism. Similarly for a small Zariski sheaf over a scheme. In this paper, we fix a category $S$ of spaces, equipped with a suitable class of morphisms which 
we call local homeomorphisms in $S$. (For example, for smooth manifolds, this class of morphisms is the class of local diffeomorphisms.) Examples of such categories of spaces are locales, sober topological spaces, various types of manifolds and schemes.

Similarly, a small stack over a space $X$ is a stack (of groupoids) $\mathcal{Z}$ over the category of open subsets of $X$. It is not reasonable to hope to construct an étalé space for $\mathcal{Z}$ whose sections over an open subset $U$ are equivalent to $\mathcal{Z}(U)$, unless each of the groupoids $\mathcal{Z}(U)$ are (equivalent to) sets, since sections of a map of spaces can only form a set. Hence, one can only find an étalé space for sheaves. If there were to be an étalé "space" associated to a stack, this "space" would need to actually be an object of a bicategory, so that sections of the map

$$
\underline{L}(\mathcal{Z}) \longrightarrow X
$$

could form a genuine nondiscrete groupoid. In this paper we show that this can be accomplished if we, instead of searching for an étalé space, look for an étalé étale stack, which we less awkwardly name the étalé realization of $\mathcal{Z}$. In fact, we extend this result to the setting of small stacks of groupoids over étale stacks, for various notions of spaces.

We define the notion of a small sheaf and stack over an étale stack in much the same way as for topological spaces, by finding an appropriate substitute for a Grothendieck site of open subsets. Sheaves over this site are what we call small sheaves over $\mathcal{X}$, and similarly for stacks. For example, if $G$ is a discrete group acting on a topological space $X$, the stacky quotient $X / / G$ is an étale topological stack, and a small sheaf over $X / / G$ is the same as a $G$-equivariant sheaf over $X$, which can be described as a space $E$ equipped with an action of $G$ and a local homeomorphism $E \rightarrow X$ which is equivariant with respect to the two $G$-actions. If $\mathcal{X}$ happens to be an orbifold, then there is an existing notion of sheaf over $\mathcal{X}$, and it agrees with the definition of a small sheaf over $\mathcal{X}$ in the sense of this paper.

Remark The reason for the adjective small is that there is also the notion of a large sheaf or stack. For example, if $X$ is a topological space, a large sheaf over $X$ is a sheaf over the induced Grothendieck site $\mathbb{T O P} / X$. Sheaves on this site are canonically equivalent to the slice category $\operatorname{Sh}(\mathbb{T} \mathbb{O P}) / X$. More generally, for any stack $\mathcal{X}$ on $\mathbb{T O P}$, there is a canonical 2-topos of large stacks over $\mathcal{X}$, which is equivalent to $\operatorname{St}(\mathbb{T O P}) / \mathcal{X}$. In other words, a large stack over $\mathcal{X}$ is simply a map $\mathcal{Y} \rightarrow \mathcal{X}$ from another stack. A small sheaf or stack over a space or stack should be thought of as algebraic data attached to that space or stack, whereas a large sheaf or stack should be thought of as a geometric object sitting over it. This distinction was highlighted by Metzler [15]. 


\subsection{Organization and main results}

Section 2 starts by briefly recalling the basic definitions of étale stacks. It is then explained how to associate to any stack a canonical topos of small sheaves in a functorial way. In case the stack in question is presented by a spatial groupoid $\mathcal{G}$, this topos is equivalent to the classifying topos $\mathcal{B G}$ as defined by Moerdijk [16]. Following Kock and Moerdijk [12], we associate to every (atlas for an) étale stack a canonical small site of definition for its topos of small sheaves. We define small stacks to be stacks over this site. We then give an abstract description of a generalized étalé space construction in this setting, which we call the étalé realization construction.

As a demonstration of the abstract machinery developed in this section, we also prove a tangential (yet highly interesting) theorem to the effect that, in some sense, topological stacks subsume Grothendieck topoi, once we replace the role of topological spaces with that of locales:

Theorem 1.1 There is a 2-adjunction

$$
\mathfrak{T o p} \underset{\mathcal{S}}{\stackrel{\text { Sh }}{\longleftarrow}} \mathfrak{L o c S t}
$$

exhibiting the bicategory of topoi (with only invertible 2-cells) as a reflective subbicategory of localic stacks (stacks coming from localic groupoids).

Section 3 aims at giving a concrete description of the abstract construction given in Section 2. For this, we choose to represent small stacks by groupoid objects in the topos of small sheaves. We then show how a generalized action groupoid construction gives us a concrete model for the étalé realization of small stacks. As a consequence, we prove:

Theorem 1.2 For any étale stack $\mathcal{X}$, there is an adjoint equivalence of 2-categories

$$
\operatorname{St}(\mathcal{X}) \underset{L}{\stackrel{\Gamma}{\leftrightarrows}} \operatorname{Et}(\mathcal{X})
$$

between small stacks over $\mathcal{X}$ and the 2 -category of étale stacks over $\mathcal{X}$ via a local homeomorphism.

Here $L$ is the étalé realization functor and $\Gamma$ is the "stack of sections" functor. We also determine which local homeomorphisms over $\mathcal{X}$ correspond to sheaves:

Theorem 1.3 A local homeomorphism $f: \mathcal{Z} \rightarrow \mathcal{X}$ over an étale stack $\mathcal{X}$ is equivalent to the étale realization of a small sheaf $F$ over $\mathcal{X}$ if and only if it is a representable map. 
Finally, in Section 4 we provide a concrete model for the "stack of sections" functor $\Gamma$ in terms of groupoid objects in the topos of small sheaves.

Acknowledgment I would like to thank André Henriques for his help and encouragement during the beginning of this project, as well as my Ph.D. advisor, Ieke Moerdijk. I would also like to thank Urs Schreiber for a helpful brain storming session and Dorette Pronk for fruitful e-mail correspondences. Finally, I would like to thank Philip Hackney for useful comments about the introduction of this paper. This research was conducted during my studies as a Ph.D. student at Utrecht University in the Netherlands. I am currently a postdoc at the Max Planck Institute for Mathematics in Bonn, Germany.

\section{Small sheaves and stacks over étale stacks}

\subsection{Conventions and notations concerning spaces and stacks}

Throughout this article, $S$ shall denote a fixed category whose objects we shall call "spaces," equipped with an appropriate class of morphisms, which we will refer to simply as local homeomorphisms. Here is a list of possibilities:

(I) Locales and local homeomorphisms.

(II) Sober topological spaces and local homeomorphisms.

(III) Any type of manifold (e.g., smooth manifolds, $C^{k}$ manifolds, analytic manifolds, complex manifolds, supermanifolds ...) with the appropriate version of local diffeomorphism, provided we remove all separation conditions. For example, manifolds will neither be assumed paracompact nor Hausdorff.

(IV) Schemes over any fixed base and Zariski local homeomorphisms. When viewed as maps of locally ringed spaces, Zariski local homeomorphisms are those maps

$$
(f, \varphi):\left(X, \mathcal{O}_{X}\right) \longrightarrow\left(Y, \mathcal{O}_{Y}\right)
$$

such that $f$ is a local homeomorphism and $\varphi: f^{*}\left(\mathcal{O}_{Y}\right) \stackrel{\sim}{\longrightarrow} \mathcal{O}_{X}$ is an isomorphism. Again, we do not impose any separation conditions.

This list need not be exhaustive. See Appendix B for a more systematic treatment. We will also sometimes argue point-set theoretically about objects of $S$, however, in all such cases, the arguments can be extended to locales and supermanifolds etc in the usual way. A morphism in $S$ will simply be called "continuous." For example, if $S$ is taken to be the category of smooth manifolds, the phrase "continuous map" will mean a smooth map and "local homeomorphism" will mean local diffeomorphism. Similarly 
for the other examples above. The reason for not imposing any separation conditions is to consider the étalé space (espace étalé) of a sheaf over a manifold, scheme etc as a manifold or scheme itself.

Definition 2.1 An $S$-groupoid is a groupoid object in $S$. For example, a topological groupoid is a groupoid object in $\mathbb{T O P}$, the category of topological spaces. Explicitly, it is a diagram

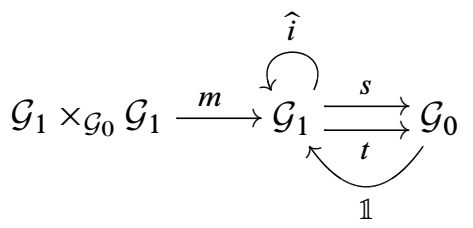

of topological spaces and continuous maps satisfying the usual axioms. Forgetting the topological structure (i.e., applying the forgetful functor from $\mathbb{T O P}$ to Set), one obtains an ordinary small groupoid. Throughout this article, we shall denote the source and target maps of a groupoid by $s$ and $t$ respectively. Similarly, a localic groupoid is a groupoid object in locales.

$S$-groupoids form a 2-category with continuous functors as 1 -morphisms and continuous natural transformations as 2-morphisms, respectively. (Recall that e.g., when $S$ is smooth manifolds, by continuous, we mean smooth.) We will denote this 2-category by $S-G p d$.

Remark A Lie groupoid is a groupoid object in smooth manifolds such that the source and target maps are submersions. Traditionally, Lie groupoids are required to have a Hausdorff object space, however, as every manifold is locally Hausdorff, any Lie groupoid in the sense we defined is Morita equivalent to one that meets this requirement. (See Definition 2.8.) We will not dwell on this issue as we will soon restrict our attention to étale groupoids.

Consider the 2-category $G p d^{S^{o p}}$ of weak presheaves in groupoids over $S$, that is contravariant (possibly weak) 2-functors from the category $S$ into the 2-category of (essentially small) groupoids $G p d .^{1}$

We recall the 2-Yoneda Lemma:

\footnotetext{
${ }^{1}$ Technically speaking, we may have to restrict ourselves to a Grothendieck universe of such spaces. If $S$ is the category of smooth manifolds, we may avoid this by replacing $\operatorname{St}(S)$ with stacks on Cartesian manifolds, i.e., manifolds of the form $\mathbb{R}^{n}$, which form a small site.
} 
Lemma 2.1 [5] If $C$ is an object of a category $\mathcal{C}$ and $\mathcal{X}$ a weak presheaf in $G p d^{\mathcal{C}^{o p}}$, then there is a natural equivalence of groupoids

$$
\operatorname{Hom}_{G p d^{\mathcal{C}^{o p}}}(C, \mathcal{X}) \simeq \mathcal{X}(C),
$$

where we have identified $C$ with its representable presheaf under the Yoneda embedding.

If $G$ is a topological group or a Lie group, then a standard example of a weak presheaf is the functor that assigns to each space the category of principal $G$-bundles over that space (this category is a groupoid). More generally, let $\mathcal{G}$ be an $S$-groupoid. Then $\mathcal{G}$ determines a weak presheaf on $S$ by the rule

$$
X \longmapsto \operatorname{Hom}_{S-G p d}\left((X)^{(\mathrm{id})}, \mathcal{G}\right)
$$

where $(X)^{(\mathrm{id})}$ is the $S$-groupoid whose object space is $X$ and has only identity morphisms. This defines an extended Yoneda 2-functor $\tilde{y}: S-G p d \rightarrow G p d^{S^{o p}}$ and we have the obvious commutative diagram

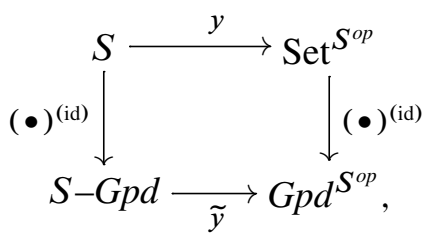

where $y$ denotes the Yoneda embedding. We denote by $[\mathcal{G}]$ the associated stack on $S, a \circ \tilde{y}(\mathcal{G})$, where $a$ is the stackification 2-functor (with respect to the open cover Grothendieck topology). $[\mathcal{G}]$ is called the stack completion of the groupoid $\mathcal{G}$.

Remark There is a notion of principal bundle for topological groupoids and Lie groupoids, and $[\mathcal{G}]$ is in fact the functor that assigns to each space the category of principal $\mathcal{G}$-bundles over that space.

Definition 2.2 A stack $\mathcal{X}$ on $\mathbb{T O P}$ is a topological stack if it is equivalent to [G] for some topological groupoid $\mathcal{G}$. A stack $\mathcal{X}$ on $M f d$, the category of smooth manifolds, is a differentiable stack if it is equivalent to $[\mathcal{G}]$ for some Lie groupoid $\mathcal{G}$. Similarly, one can define a localic stack.

Definition 2.3 An $S$-groupoid $\mathcal{G}$ is étale if its source map $s$ (and therefore also its target map $t$ ) is a local homeomorphism.

Definition 2.4 A stack $\mathcal{X}$ is on $S$ étale if it is equivalent to [G] for some étale $S$-groupoid $\mathcal{G}$. 
Definition 2.5 A morphism $f: \mathcal{Y} \rightarrow \mathcal{X}$ of stacks is said to be representable if for any map from a space $T \rightarrow \mathcal{X}$, the weak 2 -pullback $T \times \mathcal{X} \mathcal{Y}$ is (equivalent to) a space.

Remark A morphism $\varphi: \mathcal{X} \rightarrow \mathcal{Y}$ between stacks is an epimorphism (or in the language of [13], 0-connective) if it is locally essentially surjective in the following sense:

For every space $X$ and every morphism $f: X \rightarrow \mathcal{Y}$, there exists an open cover $\mathcal{U}=\left(U_{i} \hookrightarrow X\right)_{i}$ of $X$ such that for each $i$ there exists a map $\tilde{f}_{i}: U_{i} \rightarrow \mathcal{Y}$, such that the following diagram 2-commutes:

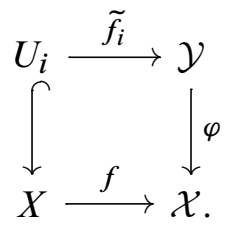

In words, this just means any map $X \rightarrow \mathcal{Y}$ from a space $X$ locally factors through $\varphi$ up to isomorphism.

Definition 2.6 An atlas for a stack $\mathcal{X}$ is a representable epimorphism $X \rightarrow \mathcal{X}$ from a space $X$.

Remark A stack $\mathcal{X}$ comes from an $S$-groupoid if and only if it has an atlas. If $X \rightarrow \mathcal{X}$ is an atlas, then $\mathcal{X}$ is equivalent to the stack completion of the groupoid $X \times \mathcal{X} X \rightrightarrows X$. Conversely, for any $S$-groupoid $\mathcal{G}$, the canonical morphism $\mathcal{G}_{0} \rightarrow[\mathcal{G}]$ is an atlas.

Definition 2.7 Let $P$ be a property of a map of spaces. It is said to be invariant under change of base if for all

$$
f: Y \longrightarrow X
$$

with property $P$, if

$$
g: Z \longrightarrow X
$$

is any representable map, the induced map

$$
Z \times_{X} Y \longrightarrow Z
$$

also has property $P$. The property $P$ is said to be invariant under restriction if this holds whenever $g$ is an open embedding. Being invariant under change of base implies being invariant under restriction. A property $P$ which is invariant under restriction is said to be local on the target if any

$$
f: Y \longrightarrow X
$$


for which there exists an open cover $\left(U_{\alpha} \rightarrow X\right)$ such that the induced map

$$
\coprod_{\alpha} U_{\alpha} \times_{X} Y \longrightarrow \coprod_{\alpha} U_{\alpha}
$$

has property $P$, must also have property $P$.

Examples of such properties are being an open map, local homeomorphism, proper map, closed map etc.

Proposition 2.1 A stack $\mathcal{X}$ over $S$ is étale if and only if it admits an étale atlas $p: X \rightarrow \mathcal{X}$, that is a representable epimorphism which is a local homeomorphism.

Proof This follows from the fact that if $\mathcal{G}$ is any $S$-groupoid, the following diagram is 2-Cartesian:

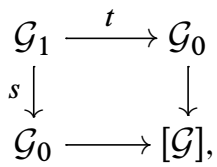

where the map $\mathcal{G}_{0} \rightarrow[\mathcal{G}]$ is induced from the canonical map $\mathcal{G}_{0} \rightarrow \mathcal{G}$.

Remark Traditionally speaking, a differentiable stack is a stack $\mathcal{X}$ equivalent to $[\mathcal{G}]$, where $\mathcal{G}$ is a Lie groupoid. This is equivalent to it having an atlas which is a representable submersion.

Definition 2.8 An internal functor $\varphi: \mathcal{H} \rightarrow \mathcal{G}$ of $S$-groupoids is a Morita equivalence if the following two properties hold:

(i) (Essentially surjective) The map $t \circ p r_{1}: \mathcal{G}_{1} \times \mathcal{G}_{0} \mathcal{H}_{0} \rightarrow \mathcal{G}_{0}$ admits local sections, where $\mathcal{G}_{1} \times{ }_{G_{0}} \mathcal{H}_{0}$ is the fibered product

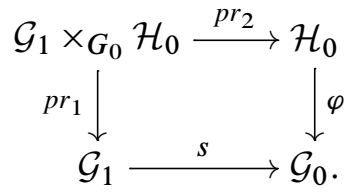

(ii) (Fully faithful) The following is a fibered product:

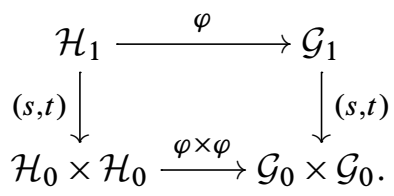

Two $S$-groupoids $\mathcal{L}$ and $\mathcal{K}$ are Morita equivalent if there is a chain of Morita equivalences $\mathcal{L} \leftarrow \mathcal{H} \rightarrow \mathcal{K}$. Also, $\mathcal{L}$ and $\mathcal{K}$ are Morita equivalent if and only if $[\mathcal{L}] \simeq[\mathcal{K}]$. 
Every internal functor $\mathcal{H} \rightarrow \mathcal{G}$ induces a map $[\mathcal{H}] \rightarrow[\mathcal{G}]$ and the induced functor

$$
\operatorname{Hom}(\mathcal{H}, \mathcal{G}) \longrightarrow \operatorname{Hom}([\mathcal{H}],[\mathcal{G}])
$$

is full and faithful, but not in general essentially surjective. However, any morphism

$$
[\mathcal{H}] \longrightarrow[\mathcal{G}]
$$

arises from a chain

$$
\mathcal{H} \leftarrow \mathcal{K} \rightarrow \mathcal{G},
$$

with $\mathcal{K} \rightarrow \mathcal{H}$ a Morita equivalence. In fact, the class of Morita equivalences admits a calculus of fractions, and stacks arising from $S$-groupoids are equivalent to the bicategory of fractions of $S$-groupoids with inverted Morita equivalences. For details, see [19].

Definition 2.9 By an étale cover of a space $X$, we mean a surjective local homeomorphism $U \rightarrow X$. In particular, for any open cover $\left(U_{\alpha}\right)$ of $X$, the canonical projection

$$
\coprod_{\alpha} U_{\alpha} \longrightarrow X
$$

is an étale cover.

Definition 2.10 Let $\mathcal{H}$ be an $S$-groupoid. If $\mathcal{U}=U \rightarrow \mathcal{H}_{0}$ is an étale cover of $\mathcal{H}_{0}$, then one can define the $\check{C}$ ech groupoid $\mathcal{H}_{\mathcal{U}}$. Its objects are $U$ and the arrows fit in the pullback diagram

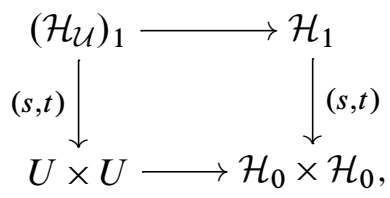

and the groupoid structure is induced from $\mathcal{H}$. There is a canonical map $\mathcal{H}_{\mathcal{U}} \rightarrow \mathcal{H}$ which is a Morita equivalence. Moreover,

$$
\operatorname{Hom}([\mathcal{H}],[\mathcal{G}]) \simeq \underset{\mathcal{U} \in \operatorname{Cov}\left(\mathcal{H}_{0}\right)}{\operatorname{holim}} \operatorname{Hom}_{S-G p d}\left(\mathcal{H}_{\mathcal{U}}, \mathcal{G}\right)
$$

where the weak 2-colimit above is taken over a suitable 2-category of étale covers. For details, see [7].

Applying (1) to the case where $[\mathcal{H}]$ is a space $X$, by the Yoneda Lemma we have

$$
[\mathcal{G}](X) \simeq \underset{\mathcal{U} \in \operatorname{Cov}(X)}{\operatorname{holim}} \operatorname{Hom}_{S-G p d}\left(X_{\mathcal{U}}, \mathcal{G}\right)
$$


Definition 2.11 Let $\mathcal{C}$ be a 2-category and $C$ an object. The slice 2-category $\mathcal{C} / C$ has as objects morphisms $\varphi: D \rightarrow C$ in $\mathcal{C}$. The morphisms are 2-commutative triangles of the form

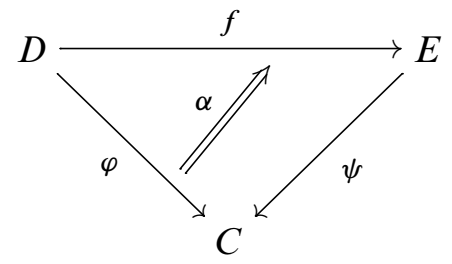

with $\alpha$ invertible. A 2-morphism between a pair of morphisms $(f, \alpha)$ and $(g, \beta)$ going between $\varphi$ and $\psi$ is a 2 -morphism in $\mathcal{C}$,

$$
\omega: f \Longrightarrow g
$$

such that the following diagram commutes:

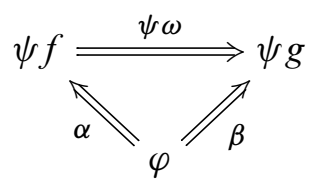

We end by a standard fact we will find useful later:

Proposition 2.2 For any stack $\mathcal{X}$ on $S$, there is a canonical equivalence of $2-$ categories $\operatorname{St}(S / \mathcal{X}) \simeq \operatorname{St}(S) / \mathcal{X}$.

The construction is as follows:

Given $\mathcal{Y} \rightarrow \mathcal{X}$ in $\operatorname{St}(S) / \mathcal{X}$, consider the stack

$$
\tilde{\mathcal{Y}}(T \rightarrow \mathcal{X}):=\operatorname{Hom}_{\mathrm{St}(S) / \mathcal{X}}(T \rightarrow \mathcal{X}, \mathcal{Y} \rightarrow \mathcal{X}) .
$$

Given a stack $\mathcal{W}$ in $\operatorname{St}(S / \mathcal{X})$, consider it as a fibered category $\int \mathcal{W} \rightarrow S / \mathcal{X}$. Then since $S / \mathcal{X} \simeq \int \mathcal{X}$ (as categories), the composition $\int \mathcal{W} \rightarrow \int \mathcal{X} \rightarrow S$ is a category fibered in groupoids presenting a stack $\widetilde{W}$ over $S$, and since the diagram

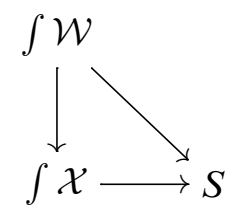

commutes, $\int \mathcal{W} \rightarrow \int \mathcal{X}$ corresponds to a map of stacks $\widetilde{\mathcal{W}} \rightarrow \mathcal{X}$.

We leave the rest to the reader. 


\subsection{Adjunctions of bicategories}

Definition 2.12 Let $\mathcal{C}$ and $\mathcal{D}$ be bicategories, and suppose that $F: \mathcal{C} \rightarrow \mathcal{D}$ and $G: \mathcal{D} \rightarrow \mathcal{C}$ are 2-functors. We say that $F$ is left adjoint to $G$ (or $G$ right adjoint to $F$ ) and write $F \dashv G$ if for each pair of objects $C \in \mathcal{C}_{0}$ and $D \in \mathcal{D}_{0}$, there exist equivalences of categories

$$
\phi_{C, D}: \operatorname{Hom}_{\mathcal{C}}(F(C), D) \stackrel{\sim}{\longrightarrow} \operatorname{Hom}_{\mathcal{D}}(C, G(D))
$$

and

$$
\psi_{C, D}: \operatorname{Hom}_{\mathcal{D}}(C, G(D)) \stackrel{\sim}{\longrightarrow} \operatorname{Hom}_{\mathcal{C}}(F(C), D),
$$

with

$$
\alpha_{C, D}: \phi_{C, D} \circ \psi_{C, D} \stackrel{\sim}{\rightleftharpoons} \mathrm{id}
$$

and

$$
\beta_{C, D}: \mathrm{id} \stackrel{\sim}{\rightleftharpoons} \psi_{C, D} \circ \phi_{C, D},
$$

such that $\phi_{\bullet, \bullet}$ and $\psi_{\bullet, \bullet}$ are weak natural transformations in each variable, and $\alpha_{\bullet}, \bullet$ and $\beta_{\bullet, \bullet}$ are modifications in each variable.

Definition 2.13 For each $C$ in $\mathcal{C}$, we have the morphism

$$
\phi_{C, F(C)}\left(\operatorname{id}_{F(C)}\right)=: \eta_{C}: C \longrightarrow G F(C) .
$$

These assemble into a weak natural transformation

$$
\eta: \mathrm{id}_{\mathcal{C}} \Longrightarrow G F
$$

called the unit of the adjunction. Similarly, for each $D$, we have the morphism

$$
\psi_{G(D), D}\left(\operatorname{id}_{G(D)}\right)=: \epsilon_{D}: F G(D) \longrightarrow D .
$$

These assemble into a weak natural transformation

$$
\epsilon: F G \Longrightarrow \mathrm{id}_{\mathcal{D}}
$$

called the counit of the adjunction.

Definition 2.14 A subbicategory $i: \mathcal{C}^{\prime} \hookrightarrow \mathcal{C}$ is called a reflective subbicategory if the inclusion $i$ has a left adjoint. Dually, if the inclusion $i$ has instead a right adjoint, it is called a coreflective subbicategory.

The following proposition is standard: 


\section{Proposition 2.3 If}

$$
\mathcal{C} \leftrightarrows \frac{G}{F} \mathcal{D}
$$

is an adjunction of bicategories with $F \dashv G$, it restricts to an equivalence between, on one hand, the full subbicategory of $\mathcal{C}$, on those objects for which the unit is an equivalence, and on the other hand, the full subbicategory of $\mathcal{D}$, for which counit is an equivalence.

Definition 2.15 If $\mathcal{C}$ is a bicategory such that for each pair of objects $C$ and $D$ in $\mathcal{C}$, $\operatorname{Hom}_{\mathcal{C}}(C, D)$ is a groupoid, $\mathcal{C}$ is a $(2,1)$-category.

Now suppose that $\mathcal{D}$ is any cocomplete $(2,1)$-category, i.e., one which has all small weak colimits, and $f: \mathcal{C} \rightarrow \mathcal{D}$ is any weak functor. Then $f$ induces a pair of adjoint functors

$$
G p d^{\mathcal{C}^{o p}} \underset{f_{*}}{\leftrightarrows} \mathcal{D},
$$

with $f^{*} \dashv f_{*}$. Explicitly

$$
f_{*}(D)(C)=\operatorname{Hom}_{\mathcal{D}}(f(C), D),
$$

and $f^{*}$ is uniquely determined up to equivalence by the fact that it is weak colimit preserving and

$$
f^{*}(y(C)) \simeq f(C) .
$$

It follows that $f^{*}$ can be given explicitly by the formula

$$
f^{*}(\mathcal{X})=\underset{C \rightarrow \mathcal{X}}{\operatorname{holim}} f(C) .
$$

The functor $f^{*}$ is the weak left Kan extension of $f$ along the Yoneda embedding $y: \mathcal{C} \rightarrow G p d^{\mathcal{C}^{o p}}$.

\subsection{Grothendieck topoi}

A concise definition of a Grothendieck topos is as follows:

Definition 2.16 A category $\mathcal{E}$ is a Grothendieck topos if it is a reflective subcategory of a presheaf category $\mathrm{Set}^{{ }^{\circ o p}}$ for some small category $\mathcal{C}$,

$$
\mathcal{E} \leftrightarrows \frac{j^{*}}{j_{*}} \operatorname{Set}^{\mathcal{C o p}^{o p}}
$$

with $j^{*} \dashv j_{*}$, such that the left adjoint $j^{*}$ preserves finite limits. From here on in, topos will mean Grothendieck topos. 
Remark It is standard that this definition is equivalent to saying that $\mathcal{E}$ is equivalent to $\operatorname{Sh}_{J}(\mathcal{C})$ for some Grothendieck topology $J$ on $\mathcal{C}$; see for example [14].

Definition 2.17 A geometric morphism from a topos $\mathcal{E}$ to a topos $\mathcal{F}$ is a an adjoint pair

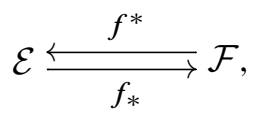

with $f^{*} \dashv f_{*}$, such that $f^{*}$ preserve finite limits. The functor $f_{*}$ is called the direct image functor, whereas the functor $f^{*}$ is called the inverse image functor.

In particular, this implies, somewhat circularly, that (2) is an example of a geometric morphism.

Topoi form a 2-category. Their arrows are geometric morphisms. If $f$ and $g$ are geometric morphisms from $\mathcal{E}$ to $\mathcal{F}$, a 2 -cell

$$
\alpha: f \Longrightarrow g
$$

is given by a natural transformation

$$
\alpha: f^{*} \Longrightarrow g^{*} \text {. }
$$

In this paper, we will simply ignore all noninvertible 2 -cells to arrive at a $(2,1)-$ category of topoi, Top.

\subsection{Locales and frames}

Locales are basically the essence of space one arrives at after liberating the definition of a topological space from the need of an underlying set. We give a more detailed review in Appendix A. Recall that the category of frames has as objects complete lattices of a certain kind and its morphisms are given by functions that preserve finite meets and arbitrary joins. The category of locales is dual to that of frames. Locales are generalized spaces and find their home in the domain of so-called pointless topology; see for example [8].

Definition 2.18 Given a topological space $X$, we denote its lattice of open subsets by $\mathcal{O}(X)$.

A continuous map $f: X \rightarrow Y$ induces a map

$$
\mathcal{O}(Y) \longrightarrow \mathcal{O}(X), \quad U \longmapsto f^{-1}(U),
$$


which is a map of frames and, hence, is a map

$$
\mathcal{O}(f): \mathcal{O}(X) \longrightarrow \mathcal{O}(Y)
$$

in the category of locales. This makes $\mathcal{O}$ into a functor

$$
\mathcal{O}: \mathbb{T O P} \longrightarrow \mathbb{L O C} \text {. }
$$

In fact, this functor has a right adjoint

$$
p t: \mathbb{L O C} \longrightarrow \mathbb{T O P} \text {. }
$$

The adjoint pair $\mathcal{O} \dashv p t$ restricts to an equivalence between sober topological spaces and locales with enough points (both "sober" and "with enough points" have a precise mathematical meaning; see Appendix A). This result is known as Stone duality. The class of sober spaces is quite large in practice. It includes many highly non-Hausdorff topological spaces such as the prime spectrum with the Zariski topology, $\operatorname{Spec}(A)$, for a commutative ring $A$.

There is a canonical full and faithful 2-functor Sh: $\mathbb{L O C} \rightarrow \mathfrak{T o p}$ which sends a locale $X$ to its topos of sheaves $\operatorname{Sh}(X)$.

\subsection{Small sheaves as a Kan extension}

Let $\mathfrak{T} \mathfrak{o p}$ denote the bicategory of Grothendieck topoi, geometric morphisms, and invertible natural transformations, as in Section 2.3. There is a canonical functor

$$
S \longrightarrow \mathfrak{T o p},
$$

which assigns each space $X$ its topos of sheaves $\operatorname{Sh}(X)$. By (weak) left-Kan extension, we obtain a 2 -adjoint pair $\mathrm{Sh} \dashv \mathcal{S}$,

$$
G p d^{S^{o p}} \underset{\mathrm{Sh}}{\stackrel{\mathcal{S}}{\leftrightarrows}} \mathfrak{T o p}
$$

where $G p d^{S^{o p}}$ denotes the bicategory of weak presheaves in groupoids. In fact, the essential image of $\mathcal{S}$ lies entirely within the bicategory of stacks over $S, \operatorname{St}(S)$, where $S$ is equipped with the standard "open cover" Grothendieck topology [2]. So, by restriction, we obtain an adjoint pair

$$
\operatorname{St}(S) \underset{\text { Sh }}{\stackrel{\mathcal{S}}{\leftrightarrows}} \mathfrak{T o p}
$$

Definition 2.19 For $\mathcal{X}$ a stack over $S$, we define the topos of small sheaves over $\mathcal{X}$ to be the topos $\operatorname{Sh}(\mathcal{X})$. 
Remark Suppose that $\mathcal{X} \simeq[\mathcal{G}]$ for some $S$-groupoid $\mathcal{G}$. Then we may consider the nerve $N(\mathcal{G})$ as a simplicial object in $S$,

$$
\mathcal{G}_{0} \longleftarrow \mathcal{G}_{1} \leftleftarrows \mathcal{G}_{2} \cdots
$$

By composition with the Yoneda embedding, we obtain a simplicial stack

$$
y \circ N(\mathcal{G}): \Delta^{o p} \longrightarrow \operatorname{St}(S) .
$$

The weak colimit of this diagram is the stack $[\mathcal{G}]$. Since Sh is a left adjoint, it follows that $\operatorname{Sh}([\mathcal{G}])$ is the weak colimit of the simplicial topos

$$
\operatorname{Sh}\left(\mathcal{G}_{0}\right) \leftleftarrows \operatorname{Sh}\left(\mathcal{G}_{1}\right) \leftleftarrows \operatorname{Sh}\left(\mathcal{G}_{2}\right) \cdots
$$

From [16], it follows that $\operatorname{Sh}([\mathcal{G}]) \simeq \mathcal{B G}$, the classifying topos of $\mathcal{G}$. We will return to a more concrete description of the classifying topos later.

For the rest of this subsection, we will assume that $S$ is the category of sober topological spaces, or locales, unless otherwise noted.

The adjoint pair $\mathrm{Sh} \dashv \mathcal{S}$ restricts to an equivalence between, on one hand, the subbicategory of $\operatorname{St}(S)$ on which the unit is an equivalence, and, on the other hand, the subbicategory of $\mathfrak{T o p}$ on which the counit is an equivalence.

Proposition 2.4 If $\mathcal{X}$ is an étale stack, then the unit is an equivalence.

Proof Let $T$ be a space, then

$$
\mathcal{S}(\operatorname{Sh}(\mathcal{X}))(T)=\operatorname{Hom}(\operatorname{Sh}(T), \operatorname{Sh}(\mathcal{X})),
$$

and the latter is the groupoid of geometric morphisms from $\operatorname{Sh}(T)$ to $\mathcal{B G}$, where $\mathcal{G}$ is some groupoid representing $\mathcal{X}$. From [16], this in turn is equivalent to $\mathcal{X}(T)$.

Let $\mathfrak{E} t$ denote the full subbicategory of $\operatorname{St}(S)$ consisting of étale stacks. Then, since the unit restricted to $\mathfrak{E t}$ is an equivalence, Sh restricted to $\mathfrak{E t}$ is 2-categorically fully faithful. We now identify its essential image.

Definition 2.20 A topos $\mathcal{E}$ is an étendue if there exists a well-supported object $E \in \mathcal{E}$ (i.e., $E \rightarrow 1$ is an epimorphism) such that the slice topos $\mathcal{E} / E$ is equivalent to $\operatorname{Sh}(X)$ for some space $X$.

Theorem 2.2 [1, Exposé iv, Exercise 9.8.2e] $A$ topos $\mathcal{E}$ is an étendue if and only if $\mathcal{E} \simeq \mathcal{B G}$ for some étale groupoid $\mathcal{G}$. 
Corollary 2.1 Sh induces an equivalence between the bicategory of étale stacks and the bicategory of étendues.

Remark This result was original proven in [19].

This corollary should be interpreted as evidence that for étale groupoids $\mathcal{G}, \operatorname{Sh}([\mathcal{G}])=\mathcal{B G}$ is the correct notion for the topos of sheaves over $[\mathcal{G}]$ since just as for spaces, morphisms between étale stacks are the same as geometric morphisms between their topoi of sheaves.

Corollary 2.2 Let $\mathcal{X} \simeq[\mathcal{G}]$ and $\mathcal{Y} \simeq[\mathcal{H}]$ be two stacks with $\mathcal{Y}$ étale. Then

$$
\operatorname{Hom}(\mathcal{X}, \mathcal{Y}) \simeq \operatorname{Hom}(\mathcal{B G}, \mathcal{B H})
$$

\subsection{Topoi as stacks}

The adjoint pair $\mathrm{Sh} \dashv \mathcal{S}$ from the previous subsection allows us also to prove another interesting result, which we shall now do, for completeness. First, we will need the concept of étale-completeness. Let $\mathcal{G}$ be a localic groupoid, and let

$$
\mathcal{G}_{0} \longrightarrow[\mathcal{G}]
$$

be the associated atlas of the localic stack $[\mathcal{G}]$. Since Sh is a left adjoint, it preserves epimorphisms, so we have an associated epimorphism

$$
p: \operatorname{Sh}\left(\mathcal{G}_{0}\right) \longrightarrow \operatorname{Sh}([\mathcal{G}]) \simeq \mathcal{B G} .
$$

By [10, C.5.3], the pullback topos

$$
\operatorname{Sh}\left(\mathcal{G}_{0}\right) \times_{\mathcal{B G}} \operatorname{Sh}\left(\mathcal{G}_{0}\right)
$$

is equivalent to sheaves on a locale $\operatorname{Sh}\left(\widehat{\mathcal{G}}_{1}\right)$ for some locale $\widehat{\mathcal{G}}_{1}$, and moreover, this canonically gives rise to a localic groupoid $\widehat{\mathcal{G}}$ with objects $\mathcal{G}_{0}$ such that the following diagram is a weak pullback:

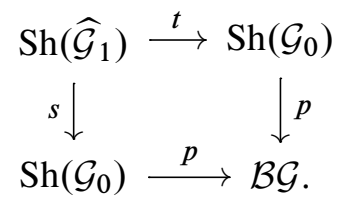

In particular, $\mathcal{B} \widehat{\mathcal{G}} \simeq \mathcal{B G}$. Notice that there is a canonical homomorphism of localic groupoids,

$$
\eta_{\mathcal{G}}: \mathcal{G} \longrightarrow \widehat{\mathcal{G}}
$$


Definition 2.21 A localic groupoid $\mathcal{G}$ is étale-complete if the diagram

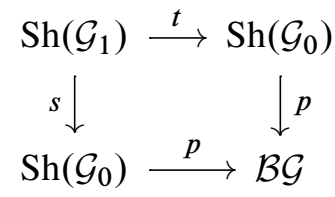

is a (weak) pullback diagram of topoi. For details, see [16].

Remark Given $x, y$ points of $\mathcal{G}_{0}$, consider the associated geometric morphisms $\hat{x}: \operatorname{Set} \rightarrow \operatorname{Sh}\left(G_{0}\right), \hat{y}: \operatorname{Set} \rightarrow \operatorname{Sh}\left(G_{0}\right)$. Then $\mathcal{G}$ is étale-complete if and only if natural isomorphisms

$$
\alpha: \hat{x}^{*} \circ p^{*} \Longrightarrow \hat{y}^{*} \circ p^{*}
$$

are in bijection with arrows $g: x \rightarrow y$ in $\mathcal{G}_{1}$.

Remark For any localic groupoid $\mathcal{G}, \widehat{\mathcal{G}}$ is étale-complete. It is called the étalecompletion of $\mathcal{G}$.

Remark Every étale groupoid is étale-complete [16].

Proposition 2.5 [16, Section 7.2] The assignment $\mathcal{G} \mapsto \widehat{\mathcal{G}}$ extends to a 2-functor

$$
\widehat{(\cdot)}: \mathbb{L O C}-G p d \longrightarrow \mathbb{E} \mathfrak{E} \mathbb{L O C}-G p d
$$

from localic groupoids to étale-complete localic groupoids, which is left adjoint to the inclusion, and with unit $\eta$.

Definition 2.22 A stack $\mathcal{X}$ over $\mathbb{L O C}$ is étale-complete if it is equivalent to [G] for some étale-complete $\mathcal{G}$.

Remark Proposition 2.4 and its proof remains valid if étale is replaced with étalecomplete.

Let $\mathfrak{E} \mathfrak{C}$ denote the full subbicategory consisting of étale-complete stacks. By the above remark, Sh restricted to $\mathfrak{E} \mathfrak{C}$ is also 2-categorically fully faithful.

Theorem 2.3 Sh induces an equivalence between the bicategory of étale-complete stacks and the bicategory Top of topoi. In particular,

$$
\mathcal{S}: \mathfrak{T o p} \longrightarrow \operatorname{St}(S)
$$

exhibits Grothendieck topoi as a reflective full subbicategory of stacks on locales. 
Proof It suffices to show that Sh is essentially surjective. Every topos is equivalent to $\mathcal{B G}$ for some localic groupoid $\mathcal{G}$ [11], and hence to $\operatorname{Sh}(\mathcal{X})$ for some localic stack $\mathcal{X}$ over locales. The result now follows from the fact that every localic groupoid $\mathcal{G}$ has an étale-completion $\widehat{\mathcal{G}}$ such that $\mathcal{B G} \simeq \mathcal{B} \widehat{\mathcal{G}}$.

Corollary 2.3 The adjunction (3) restricts to a adjunction

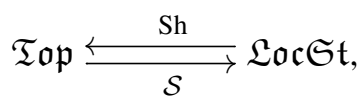

exhibiting the 2-category of topoi as a reflective subbicategory of localic stacks.

Remark In light of the fact that every topos $\mathcal{E}$ with enough points is equivalent to $\mathcal{B G}$ for some topological groupoid $\mathcal{G}$ [3], one may be tempted to claim that étalecomplete topological stacks are equivalent to topoi with enough points. However, the proof just given does not work for the topological case as a topological groupoid's étale-completion may not be a topological groupoid, but only a groupoid object in locales.

Remark Most of what has been done in this subsection caries over for smooth manifolds if we use ringed topoi rather than just topoi. In particular, the result of Pronk that étale differentiable stacks and smooth étendue are equivalent can be proven along these lines.

\subsection{The classifying topos of a groupoid}

Definition 2.23 Given an $S$-groupoid $\mathcal{H}$, a (left) $\mathcal{H}$-space is a space $E$ equipped with a moment map $\mu: E \rightarrow \mathcal{H}_{0}$ and an action map

$$
\rho: \mathcal{H}_{1} \times \mathcal{H}_{0} E \longrightarrow E
$$

where

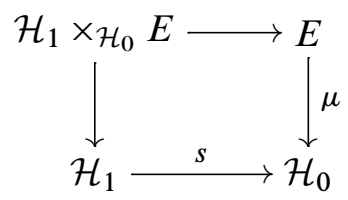

is the fibered product, such that the following conditions hold:

(i) $(g h) \cdot e=g \cdot(h \cdot e)$ whenever $e$ is an element of $E$ and $g$ and $h$ elements of $\mathcal{H}_{1}$ with domains such that the composition makes sense.

(ii) $\mathbb{1}_{\mu(e)} \cdot e=e$ for all $e \in E$.

(iii) $\mu(g \cdot e)=t(g)$ for all $g \in \mathcal{H}_{1}$ and $e \in E$. 
A map of $\mathcal{H}$-spaces is simply an equivariant map, i.e., a map

$$
(E, \mu, \rho) \longrightarrow\left(E^{\prime}, \mu^{\prime}, \rho^{\prime}\right)
$$

is map $f:(E, \mu,) \rightarrow\left(E^{\prime}, \mu^{\prime}\right)$ in $S / \mathcal{H}_{0}$ such that

$$
f(h e)=h f(e),
$$

whenever this equation makes sense.

Remark This definition extends for localic groupoids in the obvious (diagrammatic) way.

Definition 2.24 An $\mathcal{H}$-space $E$ is an $\mathcal{H}$-equivariant sheaf if the moment map $\mu$ is a local homeomorphism. The category of $\mathcal{H}$-equivariant sheaves and equivariant maps forms the classifying topos $\mathcal{B H}$ of $\mathcal{H}$.

\subsection{The small site of an étale stack}

Definition 2.25 Let $\mathcal{H}$ be an étale $S$-groupoid. Let $\operatorname{Site}(\mathcal{H})$ be the following category: The objects are the open subsets of $\mathcal{H}_{0}$. An arrow $U \rightarrow V$ is a section $\sigma$ of the sourcemap $s: \mathcal{H}_{1} \rightarrow \mathcal{H}_{0}$ over $U$ such that $t \circ \sigma: U \rightarrow V$ as a map in $S$. Composition is by the formula $\tau \circ \sigma(x):=\tau(t(\sigma(x))$.

There is a canonical functor $i: \mathcal{O}\left(\mathcal{H}_{0}\right) \hookrightarrow \operatorname{Site}(\mathcal{H})$ which sends an inclusion $U \hookrightarrow V$ in $\mathcal{O}\left(\mathcal{H}_{0}\right)$ to $\left.\mathbb{1}\right|_{U}$, where $\mathbb{1}$ is the unit map of the groupoid, and $\mathcal{O}$ is as in Definition 2.18.

This functor induces a Grothendieck pretopology on $\operatorname{Site}(\mathcal{H})$ by declaring covering families to be images under $i$ of covering families of $\mathcal{O}\left(\mathcal{H}_{0}\right)$. The Grothendieck site $\operatorname{Site}(\mathcal{H})$ equipped with the induced topology is called the small site of the groupoid $\mathcal{H}$.

Remark Given an étale stack $\mathcal{X}$ with an étale atlas $X \rightarrow \mathcal{X}$, we can describe $\operatorname{Site}(X \times \mathcal{X} X \rightrightarrows X)$ in terms of this stack and atlas. Denote the $S$-groupoid

$$
X \times \mathcal{X} X \rightrightarrows X
$$

by $\mathcal{H}$. Let $\operatorname{Site}(\mathcal{X}, X)$ denote the following category: The objects of are open subsets of $X=\mathcal{H}_{0}$ and the arrows are pairs $(f, \alpha)$, such that

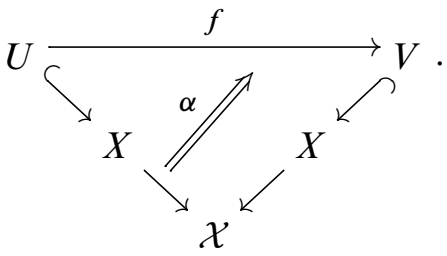


In other words, it is the full subcategory of $\operatorname{St}(S) / \mathcal{X} \simeq \operatorname{St}(S / \mathcal{X})$ (Proposition 2.2) spanned by objects of the form $U \hookrightarrow X \rightarrow \mathcal{X}$, with $U \subseteq X$ open. We claim that this category is canonically equivalent to $\operatorname{Site}(\mathcal{H})$. To see this, suppose $\sigma$ is a section of $s$ over $U$ whose image lies in $t^{-1}(V)$. We can associate to it the map

$$
\alpha(\sigma): U \longrightarrow \mathcal{H}_{1}, \quad x \longmapsto \sigma(x) .
$$

Then, letting

$$
f:=t \circ \sigma: U \longrightarrow V
$$

$\alpha(\sigma): U \rightarrow \mathcal{H}_{1}$ is a continuous natural transformation

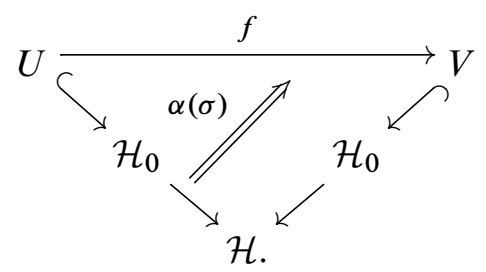

Applying stack-completion, one arrives at an arrow in $\operatorname{Site}(\mathcal{X}, X)$. Conversely, if one has a diagram of the form

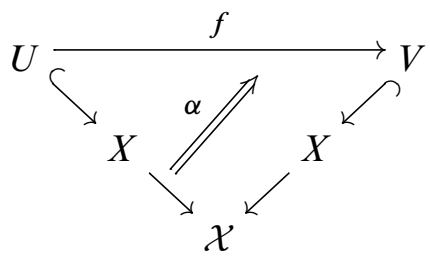

then since the canonical map

$$
\tilde{y}(\mathcal{H}) \longrightarrow[\mathcal{H}]
$$

is object-wise full and faithful, this must correspond to continuous natural transformation as in the previous diagram. But such a natural transformation, by definition, is a continuous map

$$
\alpha: U \longrightarrow \mathcal{H}_{1}
$$

such that

$$
s \circ \alpha=\operatorname{id}_{U} \quad \text { and } \quad t \circ \alpha=f .
$$

Spelling this out, one arrives at an equivalence of categories.

Definition 2.26 Given an object $U \subset \mathcal{H}_{0}$ of $\operatorname{Site}(\mathcal{H})$, the space $s^{-1}(U)$ comes equipped with a canonical left $\mathcal{H}$-action along the target map $t$. Since the target map is a local homeomorphism, this $\mathcal{H}$-space is in fact an equivariant sheaf. We denote it by $m_{U}$. 
Extend this to a functor as follows:

Given $\sigma: U \rightarrow V$ in $\operatorname{Site}(\mathcal{H})$, define a map

$$
f: s^{-1}(U) \longrightarrow s^{-1}(V)
$$

by sending

$$
x \stackrel{h}{\longrightarrow} y \quad \text { to } \quad t(\sigma(x)) \stackrel{\sigma(x)^{-1}}{\longrightarrow} x \stackrel{h}{\longrightarrow} y,
$$

which is clearly $\mathcal{H}$-equivariant. Conversely, given and $\mathcal{H}$-equivariant map

$$
f: s^{-1}(U) \longrightarrow s^{-1}(V),
$$

let $\sigma:=\left.\hat{i} \circ F \circ \mathbb{1}\right|_{U}$, where $\hat{i}$ denotes the morphism

$$
\mathcal{H}_{1} \longrightarrow \mathcal{H}_{1}
$$

which sends an arrow to its inverse. The map $\sigma$ is an object of $\operatorname{Site}(\mathcal{H})$ and it is easy to check that this defines a natural bijection

$$
\operatorname{Hom}_{\text {Site }(\mathcal{H})}(U, V) \cong \operatorname{Hom}_{\mathcal{B H}}\left(m_{U}, m_{V}\right) \text {. }
$$

Hence we get a full and faithful functor $m: \operatorname{Site}(\mathcal{H}) \rightarrow \mathcal{B H}$.

Proposition 2.6 [12] The left Kan extension of $m$ along the Yoneda embedding

$$
y: \operatorname{Site}(\mathcal{H}) \longrightarrow \operatorname{Sh}((\operatorname{Site}(\mathcal{H}))
$$

is an equivalence between the topos of sheaves for the Grothendieck site $\operatorname{Site}(\mathcal{H})$ and the classifying topos $\mathcal{B H}$.

Definition 2.27 By a small stack over an étale stack $\mathcal{X} \simeq[\mathcal{H}]$, we mean a stack $\mathcal{Z}$ over $\operatorname{Site}(\mathcal{H})$. We denote the 2 -category of small stacks over $\mathcal{X}$ by $\operatorname{St}(\mathcal{X})$.

Remark This definition does not depend on the choice of presenting groupoid since, if $\mathcal{G}$ is another groupoid such that $[\mathcal{G}] \simeq \mathcal{X}$, then

$$
\operatorname{Sh}(\operatorname{Site}(\mathcal{G})) \simeq \mathcal{B G} \simeq \mathcal{B H} \simeq \operatorname{Sh}(\operatorname{Site}(\mathcal{H}))
$$

and hence $\operatorname{St}(\operatorname{Site}(\mathcal{G})) \simeq \operatorname{St}(\operatorname{Site}(\mathcal{H}))$ by the comparison lemma for stacks [1]. A more intrinsic equivalent definition is that a small stack over $\mathcal{X}$ is a stack over the topos $\operatorname{Sh}(\mathcal{X})$ in the sense of Giraud in [6], that is a stack over $\operatorname{Sh}(\mathcal{X})$ with respect to the canonical Grothendieck topology, which in this case is generated by jointly epimorphic families. Even better, since we are dealing with étale stacks, in light of Corollary 2.1, we may instead work with the bicategory of étendues. Then, a small stack over an étendue $\mathcal{E}$ is precisely a stack over $\mathcal{E}$, with its canonical site. 


\subsection{The étalé realization of a small stack}

Recall that for a sheaf $F$ over a space $X$, the étalé space (espace étalé) is a space $E \rightarrow X$ over $X$ via a local homeomorphism (étale map), such that the sheaf of sections of $E \rightarrow X$ is isomorphic to $F$. In fact, the étalé space can be constructed for any presheaf, and the corresponding sheaf of sections is isomorphic to its sheafification. For topological spaces, as a set, $E$ is the disjoint union of the stalks of $F$ and the topology is induced by local sections.

For $S$ topological spaces or locales, this construction may be carried out abstractly as follows:

Consider the category of open subsets of $X, \mathcal{O}(X)$, where the arrows are inclusions, as in Definition 2.18. This category, equipped with its natural Grothendieck topology, is of course the site over which "sheaves over $X$ " are sheaves. There is a canonical functor $j: \mathcal{O}(X) \rightarrow S / X$ which sends an open $U \subseteq X$ to $U \hookrightarrow X$. The category $S / X$ is cocomplete, hence there is an induced adjunction

$$
\operatorname{Set}^{\mathcal{O}(X)^{o p}} \underset{L}{\leftrightarrows} S / X
$$

Here, $L$ takes a presheaf to its étalé space and $\Gamma$ takes a space $T \rightarrow X$ over $X$ to its sheaf of sections. The composite $\Gamma \circ L$ is isomorphic to the sheafification functor $a: \operatorname{Set}^{\mathcal{O}(X)} \rightarrow \mathrm{Sh}(X)$, and the image of $L$ lies completely in the subcategory $\operatorname{Et}(X)$ of $S / X$ spanned by spaces over $X$ via a local homeomorphism. When restricted to $\operatorname{Sh}(X)$ and $E t(X)$, the adjoint pair $L \dashv \Gamma$ is an equivalence of categories

$$
\operatorname{Sh}(X) \stackrel{\Gamma}{L} E t(X)
$$

This construction can be done for any category of spaces $S$ topos-theoretically as follows:

The canonical functor $j: \mathcal{O}(X) \rightarrow S / X$ produces three adjoint functors $j_{!} \dashv j^{*} \dashv j_{*}$

$$
\operatorname{Sh}(X) \rightleftarrows \operatorname{Sh}(S / X),
$$

where the Grothendieck topology on $S / X$ is induced from the open cover topology on $S$. For a sheaf $F$ over $X, j_{!}(X)=y(L(F))$, where $y$ denotes the Yoneda embedding $y: S / X \hookrightarrow \operatorname{Sh}(S / X)$.

Hence,

$$
y \circ L: \operatorname{Set}^{\mathcal{O}(X)^{o p}} \longrightarrow \operatorname{Sh}(S / X)
$$


can be identified with the left Kan extension of

$$
\mathcal{O}(X) \stackrel{j}{\longrightarrow} S / X \stackrel{y}{\longrightarrow} \operatorname{Sh}(S / X)
$$

along Yoneda.

We now turn our attention to generalizing this construction to work when both $X$ and $F$ are stacks. Let $\mathcal{H}$ be an étale groupoid and let $\mathcal{X} \simeq[\mathcal{H}]$. In light of the remark after Definition 2.25, there is a canonical fully faithful functor

$$
j_{\mathcal{H}}: \operatorname{Site}(\mathcal{H}) \longrightarrow S / \mathcal{X}
$$

which sends $U \subseteq \mathcal{H}_{0}$ to $U \hookrightarrow \mathcal{H}_{0} \rightarrow \mathcal{X}$. This produces three adjoint functors $j_{!} \dashv j^{*} \dashv j_{*}$

$$
G p d^{\operatorname{Site}(\mathcal{H})^{o p}} \rightleftarrows \operatorname{St}(S / \mathcal{X}) .
$$

We denote $j$ ! by $L$ and $j^{*}$ by $\Gamma$.

More explicitly, $j_{!}$is the weak left Kan extension of $j_{\mathcal{H}}$ along Yoneda, and

$$
\Gamma(\mathcal{Y})(U)=\operatorname{Hom}_{\mathrm{St}(S / \mathcal{X})}\left(y\left(U \hookrightarrow \mathcal{H}_{0} \rightarrow \mathcal{X}\right), \mathcal{Y}\right) .
$$

Remark Under the equivalence given in Proposition 2.2, $\mathcal{Y}$ may be viewed as stack $\overline{\mathcal{Y}}$ in $\operatorname{St}(S)$ together with a map

$$
f: \overline{\mathcal{Y}} \longrightarrow \mathcal{X}
$$

From this point of view, $\Gamma(f: \overline{\mathcal{Y}} \rightarrow \mathcal{X})$ assigns an open subset $U$ of $\mathcal{H}_{0}$ the groupoid of "sections of $f$ over $U$," which can be described explicitly as the groupoid whose objects are pairs $(\sigma, \alpha)$ which fit into a 2 -commutative diagram

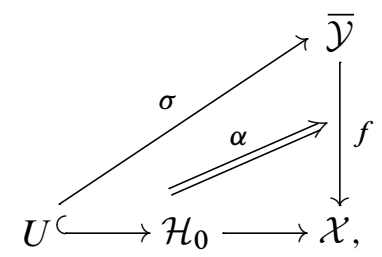

and whose morphisms $(\sigma, \alpha) \rightarrow\left(\sigma^{\prime}, \alpha^{\prime}\right)$ are 2-cells

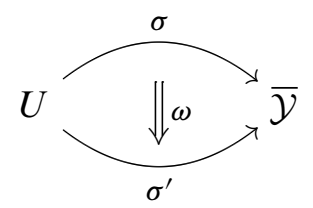


such that the following diagram commutes:

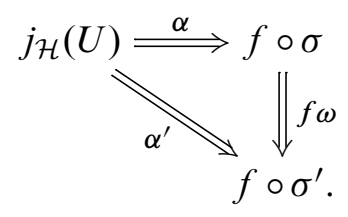

Definition 2.28 Let $\mathcal{Z}$ be a weak presheaf in groupoids over $\operatorname{Site}(\mathcal{H})$. Then $L(\mathcal{Z})$ is the étalé realization of $\mathcal{Z}$.

Proposition 2.7 Let $\mathcal{Y}$ be any stack in $\operatorname{St}(S / \mathcal{X})$. Then $\Gamma(\mathcal{X})$ is a stack.

Proof This is immediate from the fact that $\mathcal{Y}$ satisfies descent.

In fact, we can say more:

Theorem 2.4 The 2 -functor $\Gamma \circ L$ is equivalent to the stackification 2 -functor

$$
a: G p d^{\operatorname{Site}(\mathcal{H})^{o p}} \longrightarrow \operatorname{St}(\operatorname{Site}(\mathcal{H})) \simeq \operatorname{St}(\mathcal{X}) .
$$

Proof Suppose $\mathcal{Z}$ is a weak presheaf in groupoids over $\operatorname{Site}(\mathcal{H})$. Then

$$
\Gamma(\mathcal{Z})(V) \simeq L(\mathcal{Z})\left(V \hookrightarrow \mathcal{H}_{0} \rightarrow \mathcal{X}\right) .
$$

Let $G(\mathcal{Z})$ be the weak presheaf in groupoids over $S / \mathcal{X}$ given by

$$
G(\mathcal{Z}) \simeq \underset{U \rightarrow \mathcal{Z}}{\operatorname{holim}} y\left(U \hookrightarrow \mathcal{H}_{0} \rightarrow \mathcal{X}\right) .
$$

Then $\Gamma L(\mathcal{Z})(V) \simeq a(G(\mathcal{Z}))\left(V \hookrightarrow \mathcal{H}_{0} \rightarrow \mathcal{X}\right)$, where $a$ is stackification.

Note:

$$
\begin{aligned}
G(\mathcal{Z})\left(W \hookrightarrow \mathcal{H}_{0} \rightarrow \mathcal{X}\right) & \\
& \simeq \underset{U \rightarrow \mathcal{Z}}{\operatorname{holim}} \operatorname{Hom}_{\mathrm{St}(S / \mathcal{X})}\left(y\left(W \hookrightarrow \mathcal{H}_{0} \rightarrow \mathcal{X}\right), y\left(U \hookrightarrow \mathcal{H}_{0} \rightarrow \mathcal{X}\right)\right) \\
& \simeq \underset{U \rightarrow \mathcal{Z}}{\operatorname{holim}} \operatorname{Hom}_{S / \mathcal{X}}\left(W \hookrightarrow \mathcal{H}_{0} \rightarrow \mathcal{X}, U \hookrightarrow \mathcal{H}_{0} \rightarrow \mathcal{X}\right) \\
& \simeq \underset{U \rightarrow \mathcal{Z}}{\operatorname{holim}} \operatorname{Hom}_{\operatorname{Site}(\mathcal{H})}(W, U) \\
& \simeq(\underset{U \rightarrow \mathcal{Z}}{\operatorname{holim}} y(U))(W) \\
& \simeq \mathcal{Z}(W) .
\end{aligned}
$$


Given any weak presheaf in groupoids $\mathcal{W}$ over a Grothendieck site $(\mathcal{C}, J)$, we define $\mathcal{W}^{+}$by

$$
\mathcal{W}^{+}(C)=\underset{\left(C_{i} \rightarrow C\right)_{i}}{\operatorname{holim}} \underset{h o l i m}{\longleftrightarrow}\left[\prod_{i} \mathcal{W}\left(C_{i}\right) \rightrightarrows \prod_{i, j} \mathcal{W}\left(C_{i j}\right) \rightrightarrows \prod_{i, j, k} \mathcal{W}\left(C_{i j k}\right)\right]
$$

Then $a(\mathcal{W})=\mathcal{W}^{+++}$(see for instance [13, Section 6.5.3]). Now,

$G(\mathcal{Z})^{+}\left(j_{\mathcal{H}}(V)\right)$

$$
\begin{aligned}
& =\underset{\left(V_{i} \hookrightarrow V\right)_{i}}{\operatorname{holim}} \underset{h o l i m}{\longleftarrow}\left[\prod_{i} G\left(j_{\mathcal{H}}\left(V_{i}\right)\right) \rightrightarrows \prod_{i, j} G\left(j_{\mathcal{H}}\left(V_{i j}\right)\right) \rightrightarrows \prod_{i, j, k} G\left(j_{\mathcal{H}}\left(V_{i j k}\right)\right)\right] \\
& \simeq \underset{\left(V_{i} \hookrightarrow V\right)_{i}}{\operatorname{holim}} \operatorname{holim}\left[\prod_{i} \mathcal{Z}\left(V_{i}\right) \rightrightarrows \prod_{i, j} \mathcal{Z}\left(V_{i j}\right) \rightrightarrows \prod_{i, j, k} \mathcal{Z}\left(V_{i j k}\right)\right] \\
& \simeq \mathcal{Z}^{+}(V) \text {. }
\end{aligned}
$$

Hence

$$
\begin{aligned}
\Gamma L(\mathcal{Z})(V) & \simeq a(G(\mathcal{Z}))\left(V \hookrightarrow \mathcal{H}_{0} \rightarrow \mathcal{X}\right) \\
& \simeq(G(\mathcal{Z}))^{+++}\left(V \hookrightarrow \mathcal{H}_{0} \rightarrow \mathcal{X}\right) \\
& \simeq \mathcal{Z}^{+++}(V) \\
& \simeq a(\mathcal{Z})(V) .
\end{aligned}
$$

Corollary 2.4 The adjunction $L \dashv \Gamma$ restricts to an adjunction

$$
\operatorname{St}(\mathcal{X}) \underset{\widetilde{L}}{\stackrel{\widetilde{\Gamma}}{\leftrightarrows}} \operatorname{St}(S / \mathcal{X})
$$

where $\widetilde{L}$ and $\widetilde{\Gamma}$ denote the restrictions. This further restricts to an adjoint equivalence

$$
\operatorname{St}(\mathcal{X}) \underset{\bar{L}}{\stackrel{\bar{\Gamma}}{\leftrightarrows}} \mathscr{E} s s(L)
$$

between $\operatorname{St}(\mathcal{X})$ and its essential image under $L$.

The first part of Corollary 2.4 is clear. In general, a 2-adjunction restricts to an equivalence between, on one hand, those objects for which the component of the unit is an equivalence, and on the other hand, those objects for which the component of the counit is an equivalence. Hence, it suffices to prove that the essential image of $L$ is the same as the essential image of $\bar{L}$. In fact, we will prove more, namely: 
Proposition 2.8 Suppose $\mathcal{Z}$ is a weak presheaf of groupoids over $\operatorname{Site}(\mathcal{H})$. Then $L(\mathcal{Z}) \simeq L(a(\mathcal{Z})))$.

Proof $\tilde{L} \circ a$ and $L$ are both weak colimit preserving and agree on representables.

Remark If $\mathcal{X}$ is equivalent to a space $X$, then this construction generalizes the étale space construction from sheaves over $X$ to stacks over $X$ (in the ordinary sense). In the particular case when the stack over $X$ is a sheaf of sets, then its étale realization is its (Yoneda-embedded) étalé space.

\section{A concrete description of étalé realization}

The construction given for the étalé realization of a small stack over an étale stack, as of now, is rather abstract, since it is given as a weak left Kan extension. In order to work with this construction, we wish to give a more concrete description of it. To accomplish this, it is useful first to have a more concrete hold on how to represent these small stacks themselves.

For a general Grothendieck site $(\mathcal{C}, J)$, one way of representing stacks is by groupoid objects in sheaves. Given a groupoid object $\mathbb{G}$ in $\operatorname{Sh}(\mathcal{C})$, it defines a strict presheaf of groupoids by assigning an object $C$ of $\mathcal{C}$ the groupoid

$$
\operatorname{Hom}_{G p d(\operatorname{Sh}(\mathcal{C}))}\left(y(C)^{\mathrm{id}}, \mathbb{G}\right),
$$

where $y(C)^{\text {id }}$ is the groupoid object in sheaves with objects $y(C)$ and with only identity arrows, where $y$ denotes the Yoneda embedding. This strict presheaf is a sheaf of groupoids. In fact, there is an equivalence of 2-categories between groupoid objects in sheaves, and sheaves of groupoids. Moreover, every stack on $(\mathcal{C}, J)$ is equivalent to the stackification of such a strict presheaf arising from a groupoid object in sheaves. For details, see Appendix C.

In our case, we have a nice description of sheaves on $\operatorname{Site}(\mathcal{H})$, namely, it is the classifying topos $\mathcal{B H}$ of equivariant sheaves. Hence, we can model small stacks over $[\mathcal{H}]$ by groupoid objects in $\mathcal{H}$-equivariant sheaves. In the following subsection, we will describe a way to construct from a given groupoid object $\mathcal{K}$ in equivariant sheaves, an étale stack over $[\mathcal{H}]$ which will turn out to be equivalent to the étale realization of the stack over $\operatorname{Site}(\mathcal{H})$ associated to $\mathcal{K}$. 


\subsection{Generalized action groupoids}

Definition 3.1 Let $\mathcal{H}$ be any $S$-groupoid and let $\mathcal{K}$ be a groupoid object in $\mathcal{H}-$ spaces. In particular we have two $\mathcal{H}$-spaces $\left(\mathcal{K}_{0}, \mu_{0}, \rho_{0}\right)$ and $\left(\mathcal{K}_{1}, \mu_{1}, \rho_{1}\right)$ which are the underlying objects and arrows of $\mathcal{K}$. Note that the source map

$$
s:\left(\mathcal{K}_{1}, \mu_{1}, \rho_{1}\right) \longrightarrow\left(\mathcal{K}_{0}, \mu_{0}, \rho_{0}\right)
$$

and target map

$$
t:\left(\mathcal{K}_{1}, \mu_{1}, \rho_{1}\right) \longrightarrow\left(\mathcal{K}_{0}, \mu_{0}, \rho_{0}\right)
$$

are maps $s, t:\left(\mathcal{K}_{1}, \mu_{1},\right) \rightarrow\left(\mathcal{K}_{0}, \mu_{0},\right)$ in $S / \mathcal{H}_{0}$, hence $\mu_{0} \circ s=\mu_{0} \circ t=\mu_{1}$. Similarly for other structure maps.

We define an $S$-groupoid $\mathcal{H} \ltimes \mathcal{K}$ as follows:

The space of objects of $\mathcal{H} \ltimes \mathcal{K}$ is $\mathcal{K}_{0}$. An arrow from $x$ to $y$ is a pair $(h, k)$ with $h \in \mathcal{H}_{1}$ and $k \in \mathcal{K}_{1}$ such that $k: h x \rightarrow y$ (which implicitly means that $s(h)=\mu_{0}(x)$ ). We denote such an arrow pictorially as

$$
\stackrel{h}{x \rightarrow h} \stackrel{k}{\longrightarrow} y .
$$

In other words, $(\mathcal{H} \ltimes \mathcal{K})_{1}$ is the fibered product $\mathcal{H}_{1} \times_{\mathcal{H}_{0}} \mathcal{K}_{1}$ :

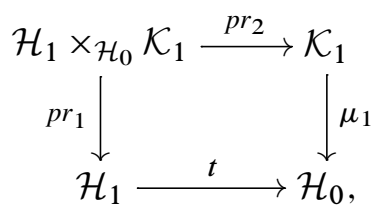

and the source and target maps are given by

$$
s(h, k)=h^{-1} s(k) \quad \text { and } \quad t(h, k)=t(k) .
$$

We need to define composition. Suppose we have two composable arrows:

$$
\underset{h}{\stackrel{h}{\rightarrow}} h x \stackrel{k}{\longrightarrow} t(k) \stackrel{h^{\prime}}{\rightarrow} h^{\prime} t(k) \stackrel{k^{\prime}}{\longrightarrow} t\left(k^{\prime}\right) .
$$

Notice that $\mu_{1}(k)=\mu_{0}(t(k))$ so that $h^{\prime}$ can act on $k$. So we get an arrow

$$
h^{\prime} \cdot k:\left(h^{\prime} h\right) x \longrightarrow h^{\prime} t(k) .
$$

We define the composition to be

$$
x \stackrel{h^{\prime} h}{\rightarrow} h^{\prime} h x \stackrel{k^{\prime}\left(h^{\prime} \cdot k\right)}{\longrightarrow} y .
$$


In other words

$$
\left(h^{\prime}, k^{\prime}\right) \circ(h, k):=\left(h^{\prime} h, k^{\prime} \circ\left(h^{\prime} \cdot k\right)\right) .
$$

The unit map $\mathcal{K}_{0} \rightarrow(\mathcal{H} \ltimes \mathcal{K})_{1}$ is given by

$$
x \longmapsto\left(\mathbb{1}_{\mu_{0}(x)}, \mathbb{1}_{x}\right),
$$

and the inverse map is given by

$$
(h, k)^{-1}:=\left(h^{-1}, h^{-1} \cdot k^{-1}\right) .
$$

Notice that if $\mathcal{K}$ is actually an $\mathcal{H}$-space $E$ considered as a groupoid object with only identity morphisms, then $\mathcal{H} \ltimes \mathcal{K}$ is the usual action groupoid $\mathcal{H} \ltimes E$. Hence, we call $\mathcal{H} \ltimes \mathcal{K}$ the generalized action groupoid of $\mathcal{K}$, or simply the action groupoid.

Remark This construction is known. It appears, for example, in [18] under the name semidirect product.

Notice that each action groupoid $\mathcal{H} \ltimes \mathcal{K}$ comes equipped with a canonical morphism $\theta_{\mathcal{K}}: \mathcal{H} \ltimes \mathcal{K} \rightarrow \mathcal{H}$ given by

$$
\left(\theta_{\mathcal{K}}\right)_{0}=\mu_{0}: \mathcal{K}_{0} \longrightarrow \mathcal{H}_{0}
$$

and

$$
\left(\theta_{\mathcal{K}}\right)_{1}=p r_{1}:(\mathcal{H} \ltimes \mathcal{K})_{1}=\mathcal{H}_{1} \times \mathcal{H}_{0} \mathcal{K}_{1} \longrightarrow \mathcal{H}_{1}
$$

The following proposition is immediate:

Proposition 3.1 If $\mathcal{H}$ is étale and $\mathcal{K}$ is a groupoid object in $\mathcal{H}$-equivariant sheaves, then $\mathcal{H} \ltimes \mathcal{K}$ is étale and the components of $\theta_{\mathcal{K}}$ are local homeomorphisms.

Remark Each groupoid object $\mathcal{K}$ in $\mathcal{H}$-spaces has an underlying $S$-groupoid $\underline{\mathcal{K}}$ and there is a canonical map $\tau_{\mathcal{K}}: \underline{\mathcal{K}} \rightarrow \mathcal{H} \ltimes \mathcal{K}$ given by the identity morphism on $\mathcal{K}_{0}$ and on arrows by

$$
k \longmapsto\left(\mathbb{1}_{\mu_{1}(k)}, k\right) .
$$

Let $(S-G p d) / \mathcal{H}$ denote the slice 2-category of $S$-groupoids over $\mathcal{H}$. We will show that the action groupoid construction

$$
\mathcal{K} \longmapsto\left((\mathcal{H} \ltimes \mathcal{K}){ }^{\theta_{\mathcal{K}}} \mathcal{H}\right)
$$

extends to a 2 -functor

$$
\mathcal{H} \ltimes: \operatorname{Gpd}(\mathcal{H} \text {-spaces }) \longrightarrow(S-G p d) / \mathcal{H} .
$$


Suppose $\varphi: \mathcal{K} \rightarrow \mathcal{L}$ is a homomorphism of groupoid objects in $\mathcal{H}$-spaces. Then we can define $\mathcal{H} \ltimes(\varphi): \mathcal{H} \ltimes \mathcal{K} \rightarrow \mathcal{H} \ltimes \mathcal{L}$ on objects as $\varphi_{0}$ and on arrows by

$$
(h, k) \longmapsto(h, \varphi(k)),
$$

which strictly commutes over $\mathcal{H}$. Finally, for $2-$ cells, given an internal natural transformation

$$
\alpha: \varphi \Longrightarrow \psi
$$

between two homomorphisms

$$
\mathcal{K} \longrightarrow \mathcal{L},
$$

$\alpha$ is in particular a map of $\mathcal{H}$-spaces $\alpha: \mathcal{K}_{0} \rightarrow \mathcal{L}_{1}$. It is easily checked that the map $\left(\tau_{\mathcal{L}}\right)_{1} \circ \alpha: \mathcal{K}_{0} \rightarrow(\mathcal{H} \ltimes \mathcal{L})_{1}$ encodes a $2-$ cell

$$
\mathcal{H} \ltimes(\alpha): \mathcal{H} \ltimes(\varphi) \Longrightarrow \mathcal{H} \ltimes(\psi),
$$

where $\tau$ is as in the remark directly proceeding Proposition 3.1 We leave it to the reader to check that this is a strict 2 -functor.

Remark This restricts to a 2 -functor

$$
\mathcal{H} \ltimes: G p d(\mathcal{B H}) \longrightarrow\left(S^{e t}-G p d\right) / \mathcal{H},
$$

where $S^{e t}$ denotes the category whose objects are spaces and arrows are all local homeomorphisms.

Let us now define a strict 2 -functor in the other direction,

$$
P:(S-G p d) / \mathcal{H} \longrightarrow \operatorname{Gpd}(\mathcal{H} \text {-spaces }) .
$$

On objects: Let $\varphi: \mathcal{G} \rightarrow \mathcal{H}$ be a map of $S$-groupoids. Consider the associated principal $\mathcal{H}$-bundle over $\mathcal{G}$. Its total space is $\mathcal{H}_{1} \times \mathcal{H}_{0} \mathcal{G}_{0}$, where

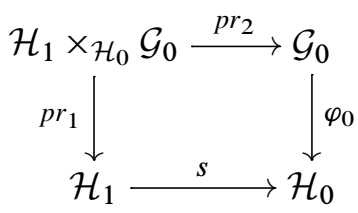

is a pullback diagram. Together with its projection $\mathrm{pr}_{2}: \mathcal{H}_{1} \times \mathcal{H}_{0} \mathcal{G}_{0} \rightarrow \mathcal{G}_{0}$, it is a right $\mathcal{G}$-space with action given by

$$
(h, x) g:=(h \varphi(g), s(g)) .
$$


We define

$$
\underline{P}(\varphi):=\left(\mathcal{H}_{1} \times_{\mathcal{H}_{0}} \mathcal{G}_{0}\right) \rtimes \mathcal{G},
$$

that is, the right action groupoid of the underlying $\mathcal{G}$-space of the associated principal bundle of $\varphi$. Since the left $\mathcal{H}$-action and right $\mathcal{G}$-action on $\mathcal{H}_{1} \times \mathcal{H}_{0} \mathcal{G}_{0}$ commute, this becomes a groupoid object in $\mathcal{H}$-spaces. Explicitly, the objects of $P(\varphi)$ are $\mathcal{H}_{1} \times \mathcal{H}_{0} \mathcal{G}_{0}$ equipped with the obvious left $\mathcal{H}$-action along $s \circ p r_{1}$ given by

$$
h^{\prime}(h, x)=\left(h^{\prime} h, x\right) .
$$

The arrows are the fibered product

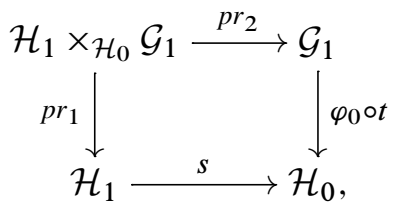

equipped with an analogously defined left $\mathcal{H}$-action along $s \circ p r_{1}$. The source and target maps are defined by

$$
s(h, g)=(h \varphi(g), s(g)) \text { and } t(h, g)=(h, t(g)) .
$$

Composition and units are defined in the obvious way.

The following proposition is immediate:

Proposition 3.2 If $\mathcal{H}$ is étale and $\varphi_{0}$ is a local homeomorphism (which implies that so is $\left.\varphi_{1}\right)$, then $P(\varphi)$ is a groupoid object in $\mathcal{B H}$.

We will now define $P$ on arrows:

Suppose we are given an arrow

$$
(f, \alpha):\left(\mathcal{G} \searrow^{\varphi} \mathcal{H}\right) \longrightarrow(\mathcal{L} \searrow \mathcal{\psi})
$$

We wish now to define an internal functor $P((f, \alpha))$. On objects, define it by:

$$
P((f, \alpha))(h, x)=\left(h \alpha(x)^{-1}, f(x)\right) .
$$

On arrows, define it by

$$
P((f, \alpha))(h, g)=\left(h \varphi(g) \alpha(s(g))^{-1} \psi(f(g))^{-1}, f(g)\right) .
$$

It is routine to verify that this defines an internal functor. 
We now define $P$ on 2-cells:

Suppose we are given a $2-$ cell $\omega:(f, \alpha) \Rightarrow\left(f^{\prime}, \alpha^{\prime}\right)$ between two maps

$$
(\mathcal{G} \searrow \mathcal{H}) \longrightarrow(\mathcal{L} \searrow \mathcal{H})
$$

Define an internal natural transformation

$$
P(\omega): P((f, \alpha)) \Longrightarrow P\left(\left(f^{\prime}, \alpha^{\prime}\right)\right), \quad P(\omega)(h, x)=(h \alpha(x), \omega(x)) .
$$

We leave it to the reader to check that $P$ is indeed a strict 2 -functor.

Lemma 3.1 There exists a natural transformation $\varepsilon$ : $\mathcal{H} \ltimes P \Rightarrow \operatorname{id}_{(S-G p d) / \mathcal{H}}$ whose components are equivalences.

Proof Given $\varphi: \mathcal{G} \rightarrow \mathcal{H}$, consider the left-action of $\mathcal{H} \times \mathcal{G}$ on

$$
\mathcal{H}_{1} \times \mathcal{H}_{0} \mathcal{G}_{0}=P(\varphi)_{0}
$$

along

$$
(h, x) \longmapsto(t(h), x)
$$

defined by

$$
(l, g) \cdot(h, x):=\left(\operatorname{lh} \varphi(g)^{-1}, t(g)\right) .
$$

Consider

$$
\theta_{P(\varphi)}:(\mathcal{H} \times \mathcal{G}) \ltimes\left(\mathcal{H}_{1} \times \mathcal{H}_{0} \mathcal{G}_{0}\right) \longrightarrow(\mathcal{H} \times \mathcal{G}),
$$

where $\theta_{P(\varphi)}$ is the canonical morphism.

By direct inspection, we see that $\mathcal{H} \ltimes P(\varphi)$ is canonically isomorphic to

$$
\tilde{\theta}_{P(\varphi)}:=p r_{1} \circ \theta_{P(\varphi)} \text {. }
$$

Consider the map

$$
\tilde{\epsilon}_{\varphi}:=p r_{2} \circ \theta_{P(\varphi)}:(\mathcal{H} \times \mathcal{G}) \ltimes\left(\mathcal{H}_{1} \times \mathcal{H}_{0} \mathcal{G}_{0}\right) \longrightarrow \mathcal{G} .
$$

Let $\xi_{\varphi}: \mathcal{H}_{1} \times \mathcal{H}_{0} \mathcal{G}_{0} \rightarrow \mathcal{H}_{1}$ be the obvious projection map. Then $\xi_{\varphi}$ is a natural isomorphism from $\varphi \circ \widetilde{\epsilon}_{\varphi}$ to $\tilde{\theta}_{P(\varphi)}$. Hence $\left(\tilde{\epsilon}_{\varphi}, \xi_{\varphi}^{-1}\right)$ is a morphism in $(S-G p d) / \mathcal{H}$ from $\tilde{\theta}_{P(\varphi)}$ to $\varphi$. It is easy to check that

$$
\epsilon: \mathcal{H} \ltimes P \Longrightarrow \operatorname{id}_{(S-G p d) / \mathcal{H}}
$$

defined by

$$
\varepsilon(\varphi)=\left(\widetilde{\varepsilon}_{\varphi}, \xi_{\varphi}^{-1}\right)
$$


is a strict natural transformations of 2-functors. It remains to see that its components consist of equivalences.

Define $\chi_{\varphi}: \mathcal{G} \rightarrow(\mathcal{H} \times \mathcal{G}) \ltimes\left(\mathcal{H}_{1} \times \mathcal{H}_{0} \mathcal{G}_{0}\right)$ on objects by

$$
\chi_{\varphi}(x)=\left(\mathbb{1}_{\varphi(x)}, x\right),
$$

and on arrows by

$$
\chi_{\varphi}(g)=\left(\left(\mathbb{1}_{\varphi(s(g))}, s(g)\right),(\varphi(g), g)\right)
$$

Then

$$
\widetilde{\varepsilon}_{\varphi} \circ \chi_{\varphi}=\mathrm{id}_{\mathcal{G}} .
$$

Note that $\tilde{\theta}_{P(\varphi)} \circ \chi_{\varphi}=\varphi$ so that $\chi_{\varphi}$ is a morphism in $(S-G p d) / \mathcal{H}$.

Define

$$
\lambda_{\varphi}: \mathcal{H}_{1} \times \mathcal{H}_{0} \mathcal{G} \longrightarrow(\mathcal{H} \times \mathcal{G}) \ltimes\left(\mathcal{H}_{1} \times \mathcal{H}_{0} \mathcal{G}_{0}\right)_{1}
$$

by

$$
\lambda_{\varphi}(h, x)=\left(\left(\mathbb{1}_{\varphi(x)}, x\right),\left(h, \mathbb{1}_{x}\right)\right) .
$$

Then $\lambda_{\varphi}$ encodes a $2-$ cell $\operatorname{id}_{\mathcal{H} \ltimes P(\varphi)} \Rightarrow \chi_{\varphi} \circ \varepsilon_{\varphi}$.

Corollary 3.1 The 2-functors

$$
\mathcal{H} \ltimes: \operatorname{Gpd}(\mathcal{H} \text {-spaces }) \longrightarrow(S-G p d) / \mathcal{H}
$$

and

$$
\mathcal{H} \ltimes: G p d(\mathcal{B H}) \longrightarrow\left(S^{e t}-G p d\right) / \mathcal{H}
$$

are bicategorically essentially surjective.

\subsection{Action groupoids are étalé realizations}

Let $\mathcal{H}$ be an étale groupoid and $\mathcal{X}$ its associated étale stack, $[\mathcal{H}]$. Let

$$
Y:\left(S^{e t}-G p d\right) / \mathcal{H} \longrightarrow \operatorname{St}(S) / \mathcal{X}
$$

be the 2 -functor which sends a groupoid $\varphi: \mathcal{G} \rightarrow \mathcal{H}$ over $\mathcal{H}$ to

$$
[\varphi]:[\mathcal{G}] \longrightarrow[\mathcal{H}]=\mathcal{X}
$$

Consider furthermore the canonical 2-functor

$$
[\cdot]_{\mathcal{B H}}: \operatorname{Gpd}(\mathcal{B H}) \longrightarrow \operatorname{St}(\operatorname{Site}(\mathcal{H}))
$$

which associates a groupoid object $\mathcal{K}$ in $\mathcal{B H}$ with its stack completion. 
Theorem 3.2 The 2 -functor $[\cdot]_{\mathcal{B H}}$ is essentially surjective and faithful (but not in general full), and the 2 -functors $\bar{L} \circ[\cdot]_{\mathcal{B H}}$ and $Y \circ \mathcal{H} \ltimes$ are equivalent.

The proof of this theorem is quite involved, so it is delayed to Appendix D.

Remark In particular, this implies that if $\mathcal{Z}$ is a small stack over $\mathcal{X}$ represented by a groupoid object $\mathcal{K}$ in $\mathcal{B H}$, then $L(\mathcal{Z}) \simeq Y(\mathcal{H} \ltimes \mathcal{K})$.

Definition 3.2 A morphism $\mathcal{Y} \rightarrow \mathcal{X}$ of étale stacks is said to be a local homeomorphism if it can be represented by a map $\varphi: \mathcal{G} \rightarrow \mathcal{H}$ of $S$-groupoids such that $\varphi_{0}$ (and hence $\left.\varphi_{1}\right)$ is a local homeomorphisms of spaces. Denote the full sub-2-category of $\operatorname{St}(S) / \mathcal{X}$ spanned by local homeomorphisms over $\mathcal{X}$ by $\operatorname{Et}(\mathcal{X})$.

In light of Theorem 3.2 and Proposition 2.8, the essential image of $L$ is precisely the local homeomorphisms over $\mathcal{X}$. Moreover, with Corollary 2.4, this implies:

\section{Corollary 3.2}

$$
\operatorname{St}(\mathcal{X}) \underset{\bar{L}}{\stackrel{\bar{\Gamma}}{\leftrightarrows}} \operatorname{Et}(\mathcal{X})
$$

is an adjoint equivalence between $\operatorname{St}(\mathcal{X})$ and local homeomorphisms over $\mathcal{X}$.

Remark Note that there is a small error in [15, top of page 44]; the construction $P_{1}$, which assigns a stack $\mathcal{Z}$ over a space $X$ an étale groupoid over $X$ via a local homeomorphism, is not functorial with respect to all maps of stacks. It is only functorial with respect to strict natural transformations of stacks, but in general, one must consider also pseudonatural transformations. The above corollary may be seen as a corrected version of this construction, in the case that $\mathcal{X}$ is a space $X$.

\subsection{The inverse image functor}

Suppose $f: \mathcal{Y} \rightarrow \mathcal{X}$ is a morphism of étale stacks. This induces a geometric morphism of 2-topoi $\operatorname{St}(\mathcal{Y}) \rightarrow \operatorname{St}(\mathcal{X})$, where by this we mean a pair of adjoint 2-functors $f^{*} \dashv f_{*}$, such that $f^{*}$ preserves finite (weak) limits. (For details about $n$-topoi, see [13, Section 6.4].) To see this, note that there is a canonical trifunctor

$$
\mathfrak{T o p} \longrightarrow 2-\mathfrak{T o p},
$$

from topoi to 2-topoi, which sends a topos $\mathcal{E}$ to the 2-topos of stacks over $\mathcal{E}$ with the canonical topology. Since

$$
\text { Sh: } \operatorname{St}(S) \longrightarrow \text { Top }
$$


is a 2 -functor, we get an induced geometric morphism

$$
\operatorname{Sh}(f): \operatorname{Sh}(\mathcal{Y}) \longrightarrow \operatorname{Sh}(\mathcal{X}),
$$

which in turn gives rise to a geometric morphism

$$
\operatorname{St}(f): \operatorname{St}(\mathcal{Y}) \longrightarrow \operatorname{St}(\mathcal{X}),
$$

after applying the trifunctor $\mathfrak{T o p} \rightarrow 2-\mathfrak{T o p}$. We denote the direct and inverse image 2-functors by $f_{*}$ and $f^{*}$.

We also get an induced geometric morphism between the 2-topoi of large stacks,

$$
\operatorname{St}(f): \operatorname{St}(S / \mathcal{Y}) \longrightarrow \operatorname{St}(S / \mathcal{X}) .
$$

This arises as the adjoint pair of slice 2 -categories

$$
\operatorname{St}(S) / \mathcal{Y} \underset{f_{*}}{\stackrel{f^{*}}{\leftrightarrows}} \operatorname{St}(S) / \mathcal{X}
$$

induced by $f$. The inverse image 2 -functor $f^{*}$ is given by pullbacks: If $\mathcal{Z} \rightarrow \mathcal{X}$ is in $\operatorname{St}(S) / \mathcal{X}$, then $f^{*}(\mathcal{Z} \rightarrow \mathcal{X})$ is given by $\mathcal{Y} \times \mathcal{X} \mathcal{Z} \rightarrow \mathcal{Y}$.

Theorem 3.3 The following diagram 2-commutes:

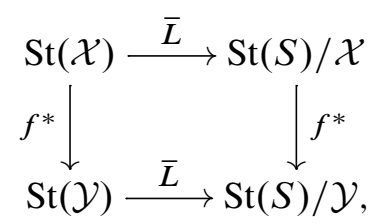

where $\bar{L}$ is as in Corollary 2.4 .

Proof As both composites $f^{*} \circ \bar{L}$ and $\bar{L} \circ f^{*}$ are weak colimit preserving, it suffices to show that they agree on representables. We fix an étale $S$-groupoid $\mathcal{H}$ such that $[\mathcal{H}] \simeq \mathcal{X}$ and choose a particular $\mathcal{G}$ such that

$$
[\mathcal{G}] \simeq \mathcal{Y}
$$

and $f=[\varphi]$ with $\varphi: \mathcal{G} \rightarrow \mathcal{H}$ an internal functor. Choose a representable sheaf $m_{U} \in \mathcal{B H}$. From [16], for any equivariant sheaf

$$
\mathcal{H} \circlearrowright E \stackrel{\mu}{\longrightarrow} \mathcal{H}_{0}
$$

$\varphi^{*}(E)$ as a sheaf over $\mathcal{G}_{0}$ is given by

$$
\mathcal{G}_{0} \times{ }_{\mathcal{H}_{0}} E \longrightarrow \mathcal{G}_{0}
$$


and has the $\mathcal{G}$-action

$$
g \cdot(x, e)=(t(g), \varphi(g) \cdot e) .
$$

Hence $\bar{L}\left(f^{*} m_{u}\right)$ is given by $Y\left(\mathcal{G} \ltimes\left(\mathcal{G}_{0} \times \mathcal{H}_{0} s^{-1}(U)\right)\right)$. Explicitly, the arrows may be described by pairs $(g, h) \in \mathcal{G}_{1} \times s^{-1}(U)$ such that

$$
s \varphi(g)=t(h) .
$$

The other composite,

$$
f^{*} \bar{L}\left(m_{u}\right)
$$

is given by

$$
[\mathcal{G}] \times{ }_{[\mathcal{H}]}\left[\mathcal{H} \ltimes s^{-1}(U)\right] \longrightarrow[\mathcal{G}] .
$$

Since the extended Yoneda 2-functor preserves all weak limits, and stackification preserves finite ones, this pullback may be computed in $S$-groupoids. Its objects are triples

$$
(z, h, \alpha) \in \mathcal{G}_{0} \times s^{-1}(U) \times \mathcal{H}_{1}
$$

such that

$$
\varphi_{0}(z) \stackrel{\alpha}{\longrightarrow} t(h) .
$$

Its arrows are quadruples

$$
\left(g, h, h^{\prime}, \alpha\right) \in \mathcal{G}_{1} \times \mathcal{H}_{1} \times s^{-1}(U) \times \mathcal{H}_{1}
$$

such that

$$
s(\varphi(g))=s(\alpha) \quad \text { and } \quad t(\alpha)=s\left(h^{\prime}\right)=t(h) .
$$

Such a quadruple is an arrow from $(s(g), h, \alpha)$ to $\left(t(g), h^{\prime} h, h^{\prime} \alpha \varphi(g)^{-1}\right)$. The projections are defined by

$$
\begin{aligned}
p r_{1}: \mathcal{G} \times \mathcal{H}\left(\mathcal{H} \ltimes s^{-1}(U)\right) & \longrightarrow \mathcal{G}, \\
(z, h, \alpha) & \longmapsto z, \\
\left(g, h^{\prime}, h, \alpha\right) & \longmapsto g,
\end{aligned}
$$

and

$$
\begin{aligned}
p r_{2}: \mathcal{G} \times \mathcal{H}\left(\mathcal{H} \ltimes s^{-1}(U)\right) & \longrightarrow \mathcal{H} \ltimes s^{-1}(U), \\
(z, h, \alpha) & \longmapsto h, \\
\left(g, h^{\prime}, h, \alpha\right) & \longmapsto\left(h^{\prime}, h\right) .
\end{aligned}
$$

We now define an internal functor

$$
\zeta: \mathcal{G} \times \mathcal{H}\left(\mathcal{H} \ltimes s^{-1}(U)\right) \longrightarrow \mathcal{G} \ltimes\left(\mathcal{G}_{0} \times \mathcal{H}_{0} s^{-1}(U)\right)
$$


on objects by

$$
(z, h, \alpha) \longmapsto\left(z, \alpha^{-1} h\right)
$$

and on arrows by

$$
\left(g, h^{\prime}, h, \alpha\right) \longmapsto\left(g, \alpha^{-1} h\right) .
$$

We define another internal functor

$$
\psi: \mathcal{G} \ltimes\left(\mathcal{G}_{0} \times \mathcal{H}_{0} s^{-1}(U)\right) \longrightarrow \mathcal{G} \times \mathcal{H}\left(\mathcal{H} \ltimes s^{-1}(U)\right)
$$

on objects as

$$
(z, h) \longmapsto\left(z, \mathbb{1}_{s(h)}, h^{-1}\right)
$$

and on arrows as

$$
(g, h)=\left(g, \mathbb{1}_{s(h)}, \mathbb{1}_{s(h)}, h^{-1}\right) .
$$

Note that $\psi$ is a left inverse for $\zeta$. We define an internal natural isomorphism

$$
\omega: \psi \circ \zeta \Longrightarrow \operatorname{id}_{\mathcal{G} \times \mathcal{H}}\left(\mathcal{H} \ltimes s^{-1}(U)\right)
$$

by

$$
\omega(z, h, \alpha)=\left(\mathbb{1}_{z}, h^{-1}, h, \alpha\right):(z, h, \alpha) \longrightarrow\left(z, \mathbb{1}_{s(h)}, h^{-1} \alpha\right)=\psi \zeta(z, h, \alpha) .
$$

As both $\zeta$ and $\psi$ commute strictly over $\mathcal{G}$, this establishes our claim.

\subsection{A classification of sheaves}

From Corollary 3.2, we know that for an étale stack $\mathcal{X}$, the 2-category of local homeomorphisms over $\mathcal{X}$ is equivalent to the 2 -category of small stacks over $\mathcal{X}$. A natural question is which objects in $E t(\mathcal{X})$ are actually sheaves over $\mathcal{X}$, as opposed to stacks, i.e., what are the 0 -truncated objects?

Theorem 3.4 A local homeomorphism $f: \mathcal{Z} \rightarrow \mathcal{X}$ over an étale stack $\mathcal{X}$ is a equivalent to $\bar{L}(F)$ for a small sheaf $F$ over $\mathcal{X}$ if and only if it is a representable map.

Proof Suppose $F$ is a small sheaf over $\mathcal{X} \simeq[\mathcal{H}]$ with $\mathcal{H}$ an étale $S$-groupoid. Denote by

$$
\bar{L}(F) \longrightarrow \mathcal{X}
$$

the map $\bar{L}(F)$. We wish to show that

$$
\underline{\bar{L}}(F) \longrightarrow \mathcal{X}
$$


is representable. It suffices to show that the 2-pullback

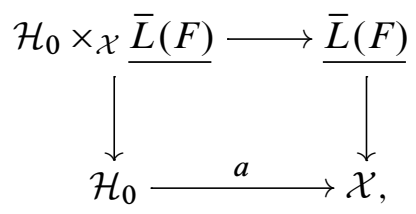

is (equivalent to) a space, where $a: \mathcal{H}_{0} \rightarrow \mathcal{X}$ is the atlas associated to $\mathcal{H}$. By Theorem 3.3, this pullback is equivalent to the total space of the étale space of the sheaf $a^{*}(F)$ over $\mathcal{H}_{0}$. Conversely, suppose $\mathcal{Z} \rightarrow \mathcal{X}$ is a representable local homeomorphism equivalent to $\bar{L}(\mathcal{W})$ for some small stack $\mathcal{W}$. Then the pullback

$$
\mathcal{H}_{0} \times \mathcal{X} \underline{\bar{L}(F)}
$$

is equivalent to a space. This implies that $a^{*}(W)$ is a sheaf of sets over $\mathcal{H}_{0}$. By definition $a^{*}(\mathcal{W})$ assigns to each open subset $U$ of $\mathcal{H}_{0}$ the groupoid $\mathcal{W}\left(m_{U}\right)$. It follows that $\mathcal{W}$ must be a sheaf.

Corollary 3.3 For an étale stack $\mathcal{X}$, the category of small sheaves over $\mathcal{X}$ is equivalent to the 2-category of representable local homeomorphisms over $\mathcal{X}$.

Remark This implies that the 2-category of representable local homeomorphisms over $\mathcal{X}$ is equivalent to its 1 -truncation.

Remark This gives a purely intrinsic definition of the topos of sheaves $\operatorname{Sh}(\mathcal{X})$. In particular, a posteriori, we could define a small stack over $\mathcal{X}$ to be a stack over this topos. We note for completeness that a site of definition of this topos is the category of local homeomorphisms $T \rightarrow \mathcal{X}$ from $T$ a space, with the induced open cover topology. This is equivalent to the category of principal $\mathcal{H}$-bundles whose moment map is a local homeomorphism.

\section{A groupoid description of the stack of sections}

Now that we have a concrete description of $\bar{L}$ in terms of groupoids, it is natural to desire a similar description for $\bar{\Gamma}$ (where $\bar{L}$ and $\bar{\Gamma}$ are as in Corollary 2.4).

Lemma 4.1 Suppose that $\varphi: T \rightarrow \mathcal{H}$ is a local homeomorphism from a space $T$, with $\mathcal{H}$ an étale groupoid. Then $\bar{\Gamma}([\varphi])$ is the equivariant sheaf $P(\varphi) \in \mathcal{B H}$, where $P$ is as in Section 3.1. 
Proof Let $m_{U}$ be a representable sheaf in $\mathcal{B H}$. Then

$$
\begin{aligned}
\Gamma([\varphi])(U) & \simeq \operatorname{Hom}\left(\bar{L}\left(m_{U}\right),[\varphi]\right) \\
& \simeq \operatorname{Hom}\left(\left[\mathcal{H} \ltimes s^{-1}(U)\right],[\varphi]\right) .
\end{aligned}
$$

Since $T$ is a sheaf, the later is in turn equivalent to

$$
\operatorname{Hom}_{G p d / \mathcal{H}}\left(\mathcal{H} \ltimes s^{-1}(U), \varphi\right) .
$$

This follows from the canonical equivalence

$$
\operatorname{Hom}\left(\tilde{y}\left(\mathcal{H} \ltimes s^{-1}(U)\right), T\right) \simeq \operatorname{Hom}\left(\left[\mathcal{H} \ltimes s^{-1}(U)\right], T\right) .
$$

In fact, this is a set, since $T$ has no arrows, so there are no natural transformations. An element of this set is the data of a groupoid homomorphism

$$
\psi: \mathcal{H} \ltimes s^{-1}(U) \longrightarrow T
$$

together with an internal natural transformation

$$
\beta: \theta_{m_{U}} \Longrightarrow \varphi \circ \psi
$$

To ease notation, let $\alpha:=\beta^{-1}$. Since $T$ is a space, $\psi_{1}$ is determined by $\psi_{0}$ by the formula

$$
\psi_{1}((h, \gamma))=\psi_{0}(\gamma)=\psi_{0}(h \gamma) .
$$

Notice that this also imposes conditions on $\psi_{0}$, namely that it is constant on orbits. The internal natural transformation is a map of spaces

$$
\alpha: s^{-1}(U) \longrightarrow \mathcal{H}_{1}
$$

such that for all $\gamma \in s^{-1}(U)$,

$$
\alpha(\gamma): \varphi \psi_{0}(\gamma) \rightarrow t(\gamma) .
$$

Because of the constraints on $\psi$, the naturality condition is equivalent to

$$
\alpha(h \gamma)=h \alpha(\gamma)
$$

This data defines a map

$$
m_{U} \longrightarrow P(\varphi)
$$

by

$$
s^{-1}(U) \longrightarrow \mathcal{H}_{1} \times \mathcal{H}_{0} T, \quad \gamma \longmapsto\left(\alpha(\gamma), \psi_{0}(\gamma)\right) .
$$

Conversely, any map $f: m_{U} \rightarrow P(\varphi)$ defines a morphism

$$
\widehat{f}: \mathcal{H} \ltimes s^{-1}(U) \longrightarrow T
$$


on objects by $p r_{2} \circ f$ (and hence determines it on arrows), and since $f$ is $\mathcal{H}$-equivariant and the $\mathcal{H}$-action on $\mathcal{H}_{1} \times \mathcal{H}_{0} T$ does not affect $T$, this map is constant on orbits. The map $f$ induces an internal natural transformation

$$
\alpha_{f}: \varphi \circ \hat{f} \longrightarrow \theta_{m_{U}}
$$

by $\alpha_{f}=p r_{1} \circ f$. This establishes a bijection

$$
\operatorname{Hom}_{G p d / \mathcal{H}}\left(\mathcal{H} \ltimes s^{-1}(U), \varphi\right) \cong \operatorname{Hom}_{\mathcal{B H}}\left(m_{U}, P(\varphi)\right) .
$$

Hence

$$
\Gamma([\varphi])(U) \simeq \operatorname{Hom}_{\mathcal{B H}}\left(m_{U}, P(\varphi)\right),
$$

so we are done by the Yoneda Lemma.

Theorem 4.2 Suppose that $\varphi: \mathcal{G} \rightarrow \mathcal{H}$ is a homomorphism of étale $S$-groupoids with $\varphi_{0}$ a local homeomorphism. Then $\bar{\Gamma}([\varphi])$ is equivalent to the stack associated to the groupoid object $P(\varphi)$ in $\mathcal{B H}$.

Proof Let $a: \mathcal{G}_{0} \rightarrow[\mathcal{G}]$ denote the atlas of the stack $[\mathcal{G}]$. There is a canonical map

$$
p: \bar{\Gamma}([\varphi] \circ a) \longrightarrow \bar{\Gamma}([\varphi]),
$$

and since $a$ is an epimorphism, it follows that $p$ is an epimorphism as well. Since $p$ is an epimorphism from a sheaf to a stack, it follows that

$$
\bar{\Gamma}([\varphi] \circ a) \times_{\bar{\Gamma}([\varphi])} \bar{\Gamma}([\varphi] \circ a) \rightrightarrows \bar{\Gamma}([\varphi] \circ a),
$$

is a groupoid object in sheaves (i.e., the classifying topos $\mathcal{B H}$ ), whose stackification is equivalent to $\bar{\Gamma}([\varphi])$. We will show that this groupoid is isomorphic to $P(\varphi)$. This isomorphism is clear on objects from the previous lemma.

Since pullbacks are computed object-wise, as a sheaf,

$$
\bar{\Gamma}([\varphi] \circ a) \times_{\bar{\Gamma}([\varphi])} \bar{\Gamma}([\varphi] \circ a)
$$

assigns $U \in \operatorname{Site}(\mathcal{H})$ the pullback groupoid

$$
\bar{\Gamma}([\varphi] \circ a)(U) \times \bar{\Gamma}_{([\varphi])(U)} \bar{\Gamma}([\varphi] \circ a)(U),
$$

which is indeed (equivalent to) a set. It is the set of pairs of objects in $\Gamma([\varphi] \circ a)(U)$ together with a morphism in $\bar{\Gamma}([\varphi])(U)$ between their images under $p(U)$.

Since for all $S$-groupoids, the induced map

$$
\operatorname{Hom}(\mathcal{L}, \mathcal{K}) \longrightarrow \operatorname{Hom}([\mathcal{L}],[\mathcal{K}])
$$


is full and faithful, we may describe this set in terms of maps of groupoids. It has the following description:

An element of $\bar{\Gamma}([\varphi] \circ a)(U) \times \bar{\Gamma}([\varphi])(U) \bar{\Gamma}([\varphi] \circ a)(U)$, can be represented by two pairs $\left(\sigma_{0}, \alpha_{0}\right)$ and $\left(\sigma_{1}, \alpha_{1}\right)$, such that for $i=0,1$,

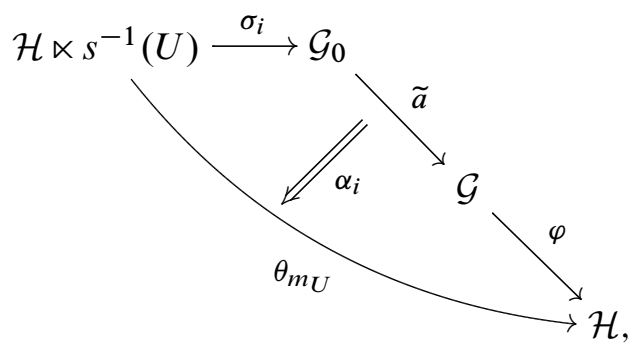

where $\tilde{a}: \mathcal{G}_{0} \rightarrow \mathcal{G}$ is the obvious map such that $[\widetilde{a}]=a$, together with a $2-$ cell

$$
\beta: \widetilde{a} \circ \sigma_{0} \Longrightarrow \widetilde{a} \circ \sigma_{1}
$$

such that the following diagram commutes:

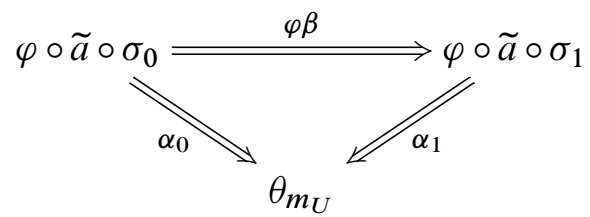

Each pair $\left(\sigma_{i}, \alpha_{i}\right)$ represents

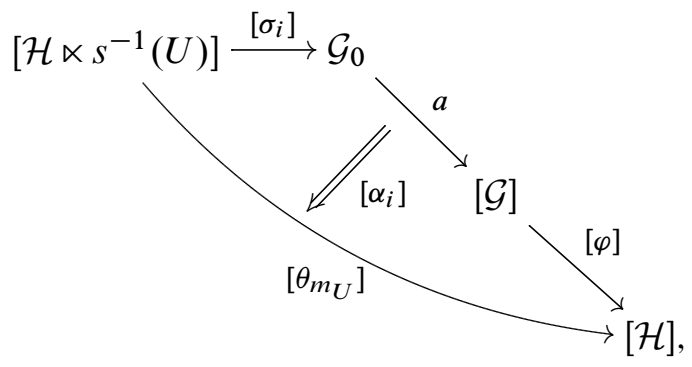

i.e., the element $\left(\left[\sigma_{i}\right],\left[\alpha_{i}\right]\right)$ of the set $\bar{\Gamma}([\varphi] \circ a)(U)$. The groupoid structure on

$$
\bar{\Gamma}([\varphi] \circ a) \times_{\bar{\Gamma}([\varphi])} \bar{\Gamma}([\varphi] \circ a) \rightrightarrows \bar{\Gamma}([\varphi] \circ a),
$$

is such that the data

$$
\left(\left(\sigma_{0}, \alpha_{0}\right),\left(\sigma_{1}, \alpha_{1}\right), \beta\right)
$$

is an arrow from $\left(\left[\sigma_{0}\right],\left[\alpha_{0}\right]\right)$ to $\left(\left[\sigma_{0}\right],\left[\alpha_{0}\right]\right)$. 
Recall that the arrows of $P(\varphi)$ are the equivariant sheaf described as the fibered product

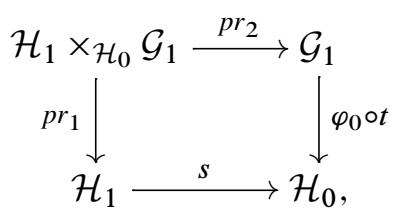

equipped with the left $\mathcal{H}$-action along $s \circ p r_{1}$ given by

$$
h \cdot(\gamma, g)=(h \gamma, g) \text {, }
$$

and that the source and target maps are given by

$$
s(h, g)=(h \varphi(g), s(g)) \text { and } t(h, g)=(h, t(g)) .
$$

Viewing the arrows of $P(\varphi)$ as a sheaf, they assign $U$ the set

$$
\operatorname{Hom}\left(m_{U}, P(\varphi)_{1}\right) \text {. }
$$

Let

$$
\pi(U): \bar{\Gamma}([\varphi] \circ a)(U) \times_{\bar{\Gamma}([\varphi])(U)} \bar{\Gamma}([\varphi] \circ a)(U) \longrightarrow \operatorname{Hom}\left(m_{U}, P(\varphi)_{1}\right)
$$

be the map that sends

$$
\zeta:=\left(\left(\sigma_{0}, \alpha_{0}\right),\left(\sigma_{1}, \alpha_{1}\right), \beta\right)
$$

to the morphism

$$
\theta(\zeta): m_{U} \longrightarrow \mathcal{H}_{1} \times \mathcal{H}_{0} \mathcal{G}_{1}, \quad \gamma \longmapsto\left(\alpha_{1}(\gamma), \beta(\gamma)\right)
$$

It is easy to check that this morphism is $\mathcal{H}$-equivariant, hence is a map in $\mathcal{B H}$. We will show that under the identification

$$
\bar{\Gamma}([\varphi] \circ a)(U) \cong P(\varphi \circ \widetilde{a})(U)=\operatorname{Hom}\left(m_{U}, P(\varphi \circ \widetilde{a})\right),
$$

$\pi(U)$ respects source and targets. Indeed, suppose we start with a triple

$$
\zeta:=\left(\left(\sigma_{0}, \alpha_{0}\right),\left(\sigma_{1}, \alpha_{1}\right), \beta\right) .
$$

By Lemma 4.1, each $\left(\sigma_{i}, \alpha_{i}\right)$ corresponds to an element of

$$
\bar{\Gamma}([\varphi] \circ a)(U),
$$

which in turn corresponds to a morphism

$$
\begin{aligned}
m_{U}=s^{-1}(U) & \longrightarrow \mathcal{H}_{1} \times{ }_{\mathcal{H}_{0}} T, \\
\gamma & \longmapsto\left(\alpha_{i}(\gamma), \sigma_{i}(\gamma)\right),
\end{aligned}
$$


in $\mathcal{B H}$. Now $\pi(U)(\zeta)$ is a map from $d_{0} \pi(U)(\zeta)$ to $d_{1} \pi(U)(\zeta)$, where we have used simplicial notation for the source and target. For each $i$, we have a map

$$
m_{U} \stackrel{\pi(U)(\zeta)}{\longrightarrow} \mathcal{H}_{1} \times \mathcal{H}_{0} \mathcal{G}_{1} \stackrel{d_{i}}{\longrightarrow} \mathcal{H}_{1} \times \mathcal{H}_{0} \mathcal{G}_{0}
$$

which we may interpret as an element of

$$
P(\varphi)_{0}(U)=P(\varphi \circ \tilde{a})(U)
$$

From (5) and the definition of the source and target map, it follows that

$$
\begin{aligned}
s \pi(U)(\zeta) & =\gamma \longmapsto s\left(\alpha_{1}(\gamma), \beta(\gamma)\right) \\
& =\gamma \longmapsto\left(\alpha_{1} \varphi \beta(\gamma), s \beta(\gamma)\right) \\
& =\gamma \longmapsto\left(\alpha_{0}(\gamma), \sigma_{0}(\gamma)\right),
\end{aligned}
$$

and

$$
\begin{aligned}
t \pi(U)(\zeta) & =\gamma \longmapsto t\left(\alpha_{1}(\gamma), \beta(\gamma)\right) \\
& =\gamma \longmapsto\left(\alpha_{1}(\gamma), t \beta(\gamma)\right) \\
& =\gamma \longmapsto\left(\alpha_{1}(\gamma), \sigma_{1}(\gamma)\right)
\end{aligned}
$$

Hence $\pi(U)$ respects the source and target. We will now show it is an isomorphism. Suppose we are given an arbitrary equivariant map

$$
\theta: m_{U} \longrightarrow \mathcal{H}_{1} \times \mathcal{H}_{0} \mathcal{G}_{1}
$$

Denote its components by

$$
\theta(\gamma)=(h(\gamma), g(\gamma))
$$

Since $\theta$ is $\mathcal{H}$-equivariant, it follows that $h$ is $\mathcal{H}$-equivariant and $g$ is $\mathcal{H}$-invariant. Now

$$
\begin{aligned}
s \circ \theta: m_{u} & \longrightarrow P(\varphi)_{0}, \\
s \theta(\gamma) & =(h(\gamma) \varphi(g(\gamma)), s(g(\gamma))),
\end{aligned}
$$

and

$$
\begin{aligned}
t \circ \theta: m_{u} & \longrightarrow P(\varphi)_{0}, \\
t \theta(\gamma) & =(h(\gamma), t(g(\gamma))) .
\end{aligned}
$$

Each of these maps correspond to an element in

$$
P(\varphi)_{0}(U) \cong \bar{\Gamma}([\varphi] \circ a)(U) .
$$


By Lemma 4.1, we know that $s \circ \theta$ corresponds to the morphism of groupoids

$$
\widehat{s \circ \theta}: \mathcal{H} \ltimes s^{-1}(U)=s^{-1}(U) \longrightarrow \mathcal{G}_{0}
$$

given on objects as

$$
\gamma \longmapsto s(g(\gamma))
$$

together with a $2-$ cell

$$
\alpha_{s \theta}:[\varphi] \circ a \circ \widehat{s \circ \theta} \Longrightarrow \theta_{m_{U}}
$$

given by

$$
\alpha_{s \theta}=p r_{1} \circ s \circ \theta \text {. }
$$

Explicitly we have:

$$
\alpha_{s \theta}(\gamma)=h(\gamma) \varphi(g(\gamma)) .
$$

Similarly, we know that $t \circ \theta$ corresponds to the morphism

$$
\widehat{t \circ \theta}: \mathcal{H} \times s^{-1}(U)=s^{-1}(U) \longrightarrow \mathcal{G}_{0}
$$

given on objects as

$$
\gamma \longmapsto t(g(\gamma))
$$

together with a 2-cell

$$
\alpha_{t \theta}:[\varphi] \circ a \circ \widehat{t \circ \theta} \Longrightarrow \theta_{m_{U}}
$$

given by

$$
\alpha_{t \theta}=p r_{1} \circ t \circ \theta
$$

and we have:

$$
\alpha_{t \theta}(\gamma)=h(\gamma)
$$

The map

$$
\beta(\theta):=p r_{2} \circ \theta: s^{-1}(U) \longrightarrow \mathcal{G}_{1}
$$

which assigns $\gamma \mapsto g(\gamma)$ encodes a natural transformation

$$
\beta(\theta): \widehat{s \circ \theta} \Longrightarrow \widehat{s \circ \theta} \text {. }
$$

Moreover, we have that

$$
\alpha_{t \theta} \varphi \beta(\gamma)=h(\gamma) \circ \varphi(g(\gamma))=\alpha s \theta(\gamma),
$$

which implies the diagram (4) commutes.

Define a map

$$
\Xi(U): \operatorname{Hom}\left(m_{U}, P(\varphi)_{1}\right) \longrightarrow \bar{\Gamma}([\varphi] \circ a)(U) \times \bar{\Gamma}_{([\varphi])(U)} \bar{\Gamma}([\varphi] \circ a)(U)
$$


which assigns the morphism $\theta: m_{U} \rightarrow P(\varphi)_{1}$ the triple

$$
\left(\left(\widehat{s \circ \theta}, \alpha_{s \theta}\right),\left(\widehat{t \circ \theta}, \alpha_{t \theta}\right), \beta(\theta)\right) \text {. }
$$

This map is clearly inverse to $\pi$. We leave it to the reader to check that $\pi(U)$ respects composition. It then follows that the groupoids in sheaves

$$
\bar{\Gamma}([\varphi] \circ a) \times \bar{\Gamma}_{([\varphi])} \bar{\Gamma}([\varphi] \circ a) \quad \text { and } \quad P(\varphi)
$$

are isomorphic.

\section{Appendix A: A brief review of locales}

In this appendix, we give a brief introduction to the theory of locales. Many ideas were taken from [9] and [10]. The concept of a locale is the essence of space one arrives at after liberating the definition of a topological space from the need of an underlying set of points. Let us recall the definition of a topological space, so that we can see how it naturally gives rise to the concept of a locale. A topological space is a set $X$ together with a subset

$$
\mathcal{O}(X) \subseteq P(X)
$$

of the power set, called the open subsets, for which the following properties hold:

(1) The intersection of finitely many opens is open.

(2) The union of an arbitrary set of opens is open.

(3) Both $X$ and the empty set $\varnothing$ are open.

Notice that (1) and (2) above make no reference to the underlying set $X$. Now, the power set $P(X)$ has much more structure than just a set. In particular, it is a lattice. Recall that a lattice is a partially ordered set $(P, \leq)$ (poset) such that for any two elements $a$ and $b$ there exists an element $a \wedge b$, called the meet of $a$ and $b$ which is the greatest element less than both $a$ and $b$, and dually there is an element $a \vee b$ which is the smallest element greater than $a$ and $b . P(X)$ is a lattice since given any two subsets $A$ and $B$, they have a meet given by

$$
A \cap B
$$

and a join given by

$$
A \cup B \text {. }
$$


Conditions (1) and (2) guarantee that $\mathcal{O}(X)$ is a sublattice of $P(X)$, and that moreover, $\mathcal{O}(X)$ has arbitrary joins, given by arbitrary unions by virtue of (2). Moreover, finite meets distribute over arbitrary joins, that is:

$$
U \cap\left(\bigcup_{\alpha} V_{\alpha}\right)=\bigcup_{\alpha} U \cap V_{\alpha},
$$

a property inherited from $P(X)$. This leads to the following definition:

Definition A.1 A frame is a lattice $\mathbb{L}$, with arbitrary joins, such that finite meets distribute over arbitrary joins.

Remark Since a frame must have arbitrary joins, it follows that it has a maximal element, 1. For a topological space, the maximal element of $\mathcal{O}(X)$ is clearly $X$. By the adjoint functor theorem, any lattice in which finite meets distribute over arbitrary joins must also have arbitrary meets. Hence it follows that a frame much also have a minimal element, 0 . In the case of a topological space $X$, the arbitrary meets cannot necessarily be computed in $P(X)$, that is, they are not always given by intersection. Indeed, for a set $\left(V_{\alpha}\right)_{\alpha}$ of opens, the meet is given by

$$
\operatorname{int}\left(\bigcap_{\alpha} V_{\alpha}\right) \text {, }
$$

the interior of the intersection. In particular, the minimal element in $\mathcal{O}(X)$ is the meet of all open subsets, i.e., the empty set $\varnothing$. Hence the analogue of (3) for a frame is that it must have a maximal and minimal element.

Now, suppose that $f: X \rightarrow Y$ is a continuous map of topological spaces. That is, the induced map

$$
P(f): P(Y) \longrightarrow P(X)
$$

which sends a subset

$$
A \subseteq Y \text { to } f^{-1}(A),
$$

carries $\mathcal{O}(Y)$ to $\mathcal{O}(X)$. Notice that $P(f)$ is more than a map of lattices, as it preserves arbitrary meets and arbitrary joins. Consequently, the induced map of lattices

$$
\mathcal{O}(f): \mathcal{O}(Y) \longrightarrow \mathcal{O}(X)
$$

preserves finite meets and arbitrary joins. (It need not preserve arbitrary meets, as these need not be given by intersection.) This leads to the following definition:

Definition A.2 A map of frames is a function $f: \mathbb{L} \rightarrow \mathbb{L}^{\prime}$ which preserves finite meets and arbitrary joins. 
Consequently, frames form a category Frm. Since maps of spaces induce maps of their associated frames in the opposite direction, if one wishes to view frames as generalized spaces, one should use their opposite category. In doing so, it is customary to give them another name. Indeed, this is the definition of a locale:

Definition A.3 The category of locales is the opposite category of Frm and is denoted by $\mathbb{L O C}$. A map of locales will be called continuous.

There is an evident functor

$$
\mathcal{O}: \mathbb{T O P} \longrightarrow \mathbb{L O C}
$$

If $X$ is a topological space, then a point of $X$ is the same thing as a morphism $* \rightarrow X$ from the one point space. Hence, this suggests a reasonable definition:

Definition A.4 A point of a locale $\mathbb{L}$ is a morphism

$$
\mathcal{O}(*) \longrightarrow \mathbb{L}
$$

The set of points is denoted by $p t(\mathbb{L})$.

Whereas this is a natural definition to make, it is important to note that in general, if $X$ is a topological space, points of $X$ may not be the same as points of $\mathcal{O}(X)$. In fact, the induced map between the points of $X$ and the points of $\mathcal{O}(X)$ is a bijection if and only if $X$ is sober. We will now give a more topological description of sobriety.

Definition A.5 Let $A \subseteq X$ be a subspace of a topological space. $A$ is reducible if it can be written as

$$
A=A_{1} \cup A_{2},
$$

with both $A_{1}$ and $A_{2}$ proper closed subsets of $A$. A subset is called irreducible if it is not reducible.

Remark Let $x \in X$. Then the closure $\overline{\{x\}}$ is an irreducible closed subset of $X$.

Definition A.6 A topological space $X$ is sober if and only if every irreducible closed subset is the closure of a unique point in $X$.

The class of sober spaces is quite large in practice. It includes all Hausdorff spaces and also many highly non-Hausdorff topological spaces such as the prime spectrum of a commutative ring, with the Zariski topology, $\operatorname{Spec}(A)$ (or more generally the underlying space of any scheme). 
Suppose that $\mathbb{L}$ is a locale. Note that

$$
\mathcal{O}(*)=\{\varnothing, *\}
$$

Define a map of sets

$$
\begin{aligned}
\varphi_{\mathbb{L}}: \mathbb{L} \longrightarrow P(p t(\mathbb{L})), \\
l \in \mathbb{L} \longmapsto\{p \in p t(\mathbb{L}) \mid \text { in Frm, } p: \mathbb{L} \longrightarrow \mathcal{O}(*) \text { satisfies } p(l)=*\} .
\end{aligned}
$$

It can be checked that $\varphi_{\mathbb{L}}$ is a map of frames, so its image defines a sublattice of $P(p t(\mathbb{L}))$. In fact, this sublattice defines a topology on the set $p t(\mathbb{L})$. This defines a functor

$$
p t: \mathbb{L O C} \longrightarrow \mathbb{T O P} \text {. }
$$

Moreover, since $\mathcal{O}(p t(\mathbb{L}))$, as a frame, is the image of $\varphi_{\mathbb{L}}$, there is a canonical induced map of locales

$$
\eta_{\mathbb{L}}: \mathcal{O}(p t(\mathbb{L})) \longrightarrow \mathbb{L}
$$

Definition A.7 A locale $\mathbb{L}$ has enough points if $\eta_{\mathbb{L}}: \mathcal{O}(p t(\mathbb{L})) \rightarrow \mathbb{L}$ is an isomorphism.

Theorem A.1 (Stone duality) The functor pt is right adjoint to $\mathcal{O}$. Moreover, this adjunction restricts to an equivalence between sober topological spaces on one hand and locales with enough points on the other.

\section{A.1: Covers and local homeomorphisms}

Let $X$ be a topological space. Then an open subset of $X$ is the same as an element of the associated locale $\mathcal{O}(X)$. This leads naturally to the following definition:

Definition A.8 Let $\mathbb{L}$ be a locale and $U$ an element of $\mathbb{L}$. Then $U$ is called an open subset of $\mathbb{L}$.

For this definition to be justified, to an element of a locale $\mathbb{L}$, there should be an associated inclusion of an open sublocale. We will make this precise. To build up our intuition, consider a topological space $X$ and let

$$
i: A \hookrightarrow X
$$

be the inclusion of any subspace. Then the corresponding map of frames is

$$
\begin{aligned}
i^{-1}: \mathcal{O}(X) & \longrightarrow \mathcal{O}(A)=\{V \in \mathcal{O}(X) \mid U \subseteq A\}, \\
U & \longmapsto A \cap U .
\end{aligned}
$$


So let $U \in \mathbb{L}$ be an element of a locale. We can define the frame

$$
\downarrow U:=\{l \in \mathbb{L} \mid l \leq U\} .
$$

By the above, if $\mathbb{L}=\mathcal{O}(X)$, this is the frame $\mathcal{O}(U)$. Now there is a frame homomorphism $\pi_{U}^{*}: \mathbb{L} \rightarrow \downarrow U$, given by the same formula, namely

$$
l \longmapsto U \wedge l \text {. }
$$

This defines a map of locales $\pi_{U}: U \rightarrow \mathbb{L}$ (where we have abused notation to identify $U$ with its locale $\downarrow U$.). This map represents the open inclusion of $U$.

Definition A.9 Let $f: \mathbb{L}^{\prime} \rightarrow \mathbb{L}$ be a map of locales. It is an open embedding if there exists an element $U \in \mathbb{L}$ and a factorization of $f$ of the form

$$
\mathbb{L}^{\prime} \stackrel{\sim}{\longrightarrow} U \stackrel{\pi_{U}}{\longrightarrow} \mathbb{L} .
$$

Notice that, viewing frames as categories (since they are in particular posets), the functor $\pi_{U}^{*}$ is right adjoint to the inclusion $\left(\pi_{U}\right) !: \downarrow U \hookrightarrow \mathbb{L}$. Hence, the element $U$ may be recovered as $\left(\pi_{U}\right)_{!}(1)$. It follows that if $f: \mathbb{L}^{\prime} \rightarrow \mathbb{L}$ is an open embedding, then its associated map of frames $f^{*}: \mathbb{L} \rightarrow \mathbb{L}^{\prime}$ has a left adjoint $f_{!}$and $f_{!}(1)$ is the element of $\mathbb{L}$ corresponding to its open image. This allows us to make sense of an open covering:

Definition A.10 A family of open embeddings

$$
\left(f_{\alpha}: \mathbb{L}_{\alpha} \longrightarrow \mathbb{L}\right)
$$

is an open covering if

$$
\bigvee_{\alpha}\left(f_{\alpha}\right) !(1)=1
$$

Definition A.11 A map of locales $f: \mathbb{L}^{\prime} \rightarrow \mathbb{L}$ is a local homoeomorphism if there exists an open covering $\left(i_{\alpha}: U_{\alpha} \rightarrow \mathbb{L}^{\prime}\right)_{\alpha}$ of $\mathbb{L}^{\prime}$ such that for each $\alpha$, the composite

$$
U_{\alpha} \stackrel{i_{\alpha}}{\longrightarrow} \mathbb{L}^{\prime} \stackrel{f}{\longrightarrow} \mathbb{L}
$$

is an open embedding. A local homeomorphism is said to be surjective if the family

$$
\left(U_{\alpha} \stackrel{i_{\alpha}}{\longrightarrow} \mathbb{L}^{\prime} \stackrel{f}{\longrightarrow} \mathbb{L}\right)_{\alpha}
$$

is an open covering of $\mathbb{L}$.

Each locale carries a canonical Grothendieck pretopology on its underlying poset: 
Definition A.12 Declare a collection of morphisms $\left(U_{\alpha} \rightarrow U\right)_{\alpha}$ in $\mathbb{L}$, in other words, a collection of objects $U_{\alpha}$ such that

$$
U_{\alpha} \leq U
$$

to be a covering family, if and only if $\bigvee_{\alpha} U_{\alpha}=U$. Note that this is equivalent to demanding that the induced maps $\left(U_{\alpha} \rightarrow U\right)$ form an open covering. The resulting Grothendieck topology on $\mathbb{L}$ is called the open cover topology. The associated topos of sheaves will simply be denoted by $\operatorname{Sh}(\mathbb{L})$ and will be called the topos of small sheaves on $\mathbb{L}$.

Proposition A.1 [14] The assignment $\mathbb{L} \mapsto \operatorname{Sh}(\mathbb{L})$ extends to a full and faithful 2-functor

$$
\text { Sh: } \mathbb{L O C} \longrightarrow \text { Top. }
$$

Corollary A.1 The induced 2-functor

$$
\text { Sh: } \mathbb{T O P} \longrightarrow \text { Top }
$$

is full and faithful when restricted to sober topological spaces.

Let $\mathbb{L}$ be a locale and suppose $U$ is an open subset. Suppose that we have a commutative diagram of posets and functors

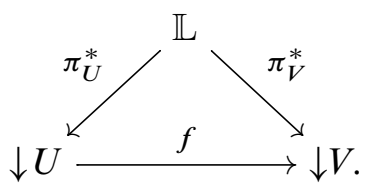

Then for any $l \in \downarrow U$,

$$
f(l)=f\left(\pi_{U}^{*}(l)\right)=\pi_{V}^{*}(l)=l \wedge V .
$$

For $f$ to be a map of frames, $f$ must preserve finite meets and arbitrary joins. In particular, it must preserve the maximal element 1 . This means one must have

$$
f(U)=U \wedge V=V,
$$

which is equivalent to demanding $V \leq U$. Moreover, one can check that if $V \leq U$, that $f$ defined as above is a map of frames. The following proposition is immediate:

Proposition A.2 Let $\mathbb{L}$ be a locale. Then the canonical functor

$$
\begin{aligned}
& \mathbb{L} \stackrel{\chi}{\longrightarrow} \mathbb{L} \mathbb{O} \mathbb{C} / \mathbb{L}, \\
& U \longmapsto \pi_{U}: U \rightarrow \mathbb{L},
\end{aligned}
$$

is fully faithful. Its essential image is the subcategory spanned by open embeddings. 
For any locale $\mathbb{L}$, the category of locales over $\mathbb{L}, \mathbb{L O C} / \mathbb{L}$, is cocomplete, so one may form the left Kan extension $L$ of $\chi$ with respect to the Yoneda embedding

$$
y: \mathbb{L} \longleftrightarrow \mathrm{Set}^{\mathbb{L}^{o p}} \text {. }
$$

$L$ comes with a right adjoint $\Gamma$ :

$$
\operatorname{Set}^{\mathbb{L}^{o p}} \stackrel{\Gamma}{\longleftarrow} \mathbb{L} \mathbb{O C} / \mathbb{L}
$$

The functor $\Gamma$ sends a map

$$
\mathbb{L}^{\prime} \longrightarrow \mathbb{L}
$$

of locales to the induced presheaf of sections of this map. This presheaf is easily seen to be a sheaf. Moreover, one has the following classical theorem:

Theorem A.2 The adjunction

$$
\mathrm{Set}^{\mathbb{L}^{o p}} \stackrel{\Gamma}{\longleftarrow} \mathbb{L} \mathbb{O C} / \mathbb{L}
$$

restricts to an adjoint equivalence

$$
\operatorname{Sh}(\mathbb{L}) \underset{L}{\stackrel{\Gamma}{\leftrightarrows}} E t(\mathbb{L})
$$

where $E t(\mathbb{L})$ is the subcategory of $\mathbb{L} \mathbb{O} \mathbb{C} / \mathbb{L}$ spanned by local homeomorphisms over $L$.

The functor $L$ associates to each sheaf $F$ on $\mathbb{L}$ a locale

$$
L F: \underline{L F} \longrightarrow \mathbb{L}
$$

over $\mathbb{L}$ via a local homeomorphism, called the étalé space of $F$. F can be recovered as the sheaf of sections of $L F$.

\section{Appendix B: Categories of spaces}

We now formalize exactly what properties are needed of a category of spaces for the results of this paper to apply to it. For a category $S$ to be considered a category of spaces, it will need to be equipped with a functor

$$
U: S \longrightarrow \mathbb{L O C}
$$

and a distinguished class of morphisms which we will call $S$-local homeomorphisms. 
Definition B.1 Let $U: S \rightarrow \mathbb{L O C}$ be a category over $\mathbb{L O C}$ and let $S^{e t}$ be a subcategory of $S$, on the same objects, such that for every

$$
f: X \longrightarrow T
$$

in $S^{e t}, U(f)$ is a local homeomorphism of locales. $U$ induces a functor

$$
U^{e t}: S^{e t} \longrightarrow \mathbb{L O C} \mathbb{C}^{e t},
$$

where $\mathbb{L O C} \mathbb{C}^{e t}$ denotes the category of locales and local homeomorphisms. $S^{e t}$ is called admissible if the following conditions hold:

(1) Every isomorphism in $S$ is in $S^{e t}$.

(2) If $f$ and $g$ are composable arrows of $S$ and any two of $f, g$ and $f g$ are in $S^{e t}$, then all three are.

(3) $S$ has pullbacks along morphisms in $S^{e t}, S^{e t}$ is stable under these and $U$ preserves these pullbacks.

(4) The canonical functor $S^{e t} \rightarrow S$ preserves coequalizers.

(5) If $f: U T \rightarrow U Z$ is a local homeomorphism of locales and there exists a family of morphisms in $S^{e t}$

$$
\left(T_{\alpha} \longrightarrow T\right)_{\alpha}
$$

such that the induced morphism

$$
\coprod_{\alpha} U T_{\alpha} \longrightarrow U T
$$

is a surjective local homeomorphism of locales, and each composite

$$
U T_{\alpha} \longrightarrow U T \longrightarrow U Z
$$

is equal to $U\left(\varphi_{\alpha}\right)$ for some $\varphi_{\alpha}$ in $S$, then there exists a morphism $\varphi: T \rightarrow Z$ in $S$ such that $U(\varphi)=f$.

(6) The induced functor

$$
U^{e t}: S^{e t} \longrightarrow \mathbb{L O C} \mathbb{C}^{e t}
$$

is faithful and locally an equivalence in the following sense: For every object $T \in S$, the induced functor

$$
U_{T}^{e t}: S^{e t} / T \longrightarrow \mathbb{L O C} \mathbb{C}^{e t} / U T
$$

is an equivalence of categories.

If $S^{e t}$ is admissible, morphisms in $S^{e t}$ are called $S$-local homeomorphisms. 
Definition B.2 Let $U: S \rightarrow \mathbb{L O C}$ be a category over $\mathbb{L O C}$ with an admissible subcategory $S^{e t}$ of $S$-local homeomorphisms. Then $S$ is called a category of spaces if the following conditions hold:

(a) $S$ has and $U$ preserves finite coproducts.

(b) If $\varphi: U T \rightarrow X$ is a surjective local homeomorphism, then there exists a $Z$ in $S$ such that $U(Z) \cong X$.

If $S$ is a category of spaces, we will often refer to objects of $S$ simply as spaces, morphisms as continuous and $S$-local homeomorphisms as local homeomorphisms.

The following is a nonexhaustive list of examples of categories of spaces in the sense of Definition B.2. In each case, the functor $U$ is obvious, so shall be omitted.

(I) Locales and local homeomorphisms.

(II) Sober topological spaces and local homeomorphisms.

(III) Any type of manifold (e.g., smooth manifolds, $C^{k}$ manifolds, analytic manifolds, complex manifolds, supermanifolds ...) with the appropriate version of local diffeomorphism, provided we remove all separation conditions. For example, manifolds will neither be assumed paracompact nor Hausdorff.

(IV) Schemes over any fixed base and Zariski local homeomorphisms. When viewed as maps of locally ringed spaces, Zariski local homeomorphisms are those maps

$$
(f, \varphi):\left(X, \mathcal{O}_{X}\right) \longrightarrow\left(Y, \mathcal{O}_{Y}\right)
$$

such that $f$ is a local homeomorphism and $\varphi: f^{*}\left(\mathcal{O}_{Y}\right) \stackrel{\sim}{\longrightarrow} \mathcal{O}_{X}$ is an isomorphism.

Remark By the conventions of Definition B.2, in this paper, if $S$ is taken to be, for example, the category of smooth manifolds, the phrase "continuous map" will mean a smooth map and "local homeomorphism" will mean local diffeomorphism. Similarly for the other examples above.

Remark In all of the examples listed, $S$ has and $U$ preserves arbitrary coproducts. We see no reason to impose this, however it does make the theory a bit nicer.

We now give some basic consequences of the definition.

Since $U_{T}^{e t}$ is an equivalence by (6), we may pick for each $T$ a left adjoint $\psi_{T}$ making the pair

$$
\psi_{T} \dashv U_{T}^{e t}
$$


an adjoint equivalence. Let us fix such a $\psi_{T}$ for each $T$ once and for all. Let

$$
\pi_{T}: S^{e t} / T \longrightarrow S^{e t}
$$

be the forgetful functor. By convention, if

$$
V \hookrightarrow U T
$$

is an open subset, then we will often refer to $\pi_{T} \psi_{T}(V \hookrightarrow U T)$ as an open subset of $T$ and denote it again by $V$. Any morphism isomorphic to the inclusion of an open subset is said to be an open embedding. It easily follows then that for any local homeomorphism in $S, f: X \rightarrow T$, there exists an open covering family (in the sense of Definition B.3)

$$
\left(V_{\alpha} \longrightarrow X\right)
$$

of $X$ such that each composite

$$
V_{\alpha} \longrightarrow X \longrightarrow T
$$

is an open embedding.

Let $f: T^{\prime} \rightarrow T$ and $g: T^{\prime \prime} \rightarrow T$ be objects in $S^{e t} / T$. Then by (3), $f \times g$ exists and is given by the canonical map

$$
T^{\prime} \times_{T} T^{\prime \prime} \longrightarrow T
$$

In particular, $S^{e t}$ has pullbacks and they are preserved and reflected by the canonical functor

$$
S^{e t} \longrightarrow S
$$

By (6), $U_{T}^{e t}$ is an equivalence, hence $U(f \times g)=U(f) \times U(g)$ in $\mathbb{L O C} \mathbb{C}^{e t} / U T$. Since the canonical diagram

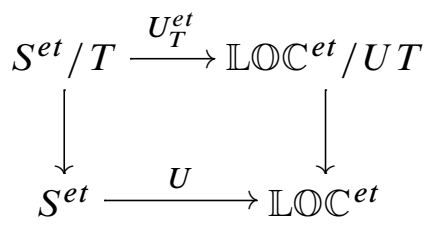

commutes, it follows that

$$
U\left(T^{\prime} \times_{T} T^{\prime \prime}\right)=U T^{\prime} \times_{U T} U T^{\prime \prime} .
$$

This shows that $U^{e t}: S^{e t} \rightarrow \mathbb{L O C} \mathbb{C}^{e t}$ preserves and reflects pullbacks. 
Definition B.3 We say a collection of local homeomorphisms $\left(T_{\alpha} \rightarrow T\right)_{\alpha}$ in $S$ is a covering family of local homeomorphisms if the induced morphism

$$
\coprod_{\alpha} U T_{\alpha} \longrightarrow U T
$$

is a surjective local homeomorphism of locales. The family is called a open covering family if each map

$$
U T_{\alpha} \longrightarrow U T
$$

is an open embedding.

Proposition B.1 If $S$ is a category of spaces, by either declaring covering families to be covering families of local homeomorphisms, or open covering families (as in Definition B.3), one gets a Grothendieck pretopology on $S$.

Proof By (1), every isomorphism is a cover. The pullback of a cover is readily seen to be a cover by virtue of (3). Transitivity of covers follows from the fact that the composite of two surjective local homeomorphisms of locales is again a surjective local homeomorphism.

Definition B.4 Both of these pretopologies induce the same Grothendieck topology, which we will call the open cover topology on $S$.

Definition B.5 The functor

$$
U: S \longrightarrow \mathbb{L} \mathbb{O C}
$$

canonically assigns each space $T$ the topos $\operatorname{Sh}(U T)$. This is called the topos of small sheaves on $T$. By abuse of notation, we will denote $\operatorname{Sh}(U T)$ by $\operatorname{Sh}(T)$.

Remark Denote by $\mathcal{O}(T)$ the essential image of

$$
\mathcal{O}(U T) \stackrel{\chi}{\longrightarrow} \mathbb{L} \mathbb{O} \mathbb{C}^{e t} / U T \stackrel{\psi_{T}}{\longrightarrow} S^{e t} / T .
$$

The open cover topology on $S^{e t}$ induces a Grothendieck topology on $\mathcal{O}(T)$ such that

$$
\operatorname{Sh}(\mathcal{O}(T)) \simeq \operatorname{Sh}(U T)
$$

Example 1 If $S$ is topological spaces, then for a space $X, \operatorname{Sh}(X)$ is the ordinary topos of sheaves on $X$. If $S$ is schemes, then for a scheme $X, \operatorname{Sh}(X)$ is the topos of sheaves on the small Zariski site of $X$. 
Combining (6) and Theorem A.2, we see that for each $T$ there is a canonical equivalence

$$
E t(T):=S^{e t} / T \simeq \mathbb{L O C} \mathbb{C}^{e t} / U T=E t(U T) \simeq \operatorname{Sh}(T) .
$$

Suppose that $F$ is a sheaf over $U T$ and that $\tilde{L F}: \underline{\tilde{L F}} \rightarrow U T$ is its étale space. Under the equivalence $U_{T}^{e t}$, this corresponds to a local homeomorphism

$$
L F: \underline{L F} \longrightarrow T
$$

in $S$. Let

$$
V \hookrightarrow T
$$

be an open subset.

Proposition B.2 The canonical map

$$
\Gamma(L F, V) \longrightarrow \Gamma(\tilde{L F}, U V)
$$

from sections of $L F$ over $V$ in $S$ to sections of $\tilde{L F}$ over $U V$ in $\mathbb{L O C}$ is a bijection.

Proof Since $U^{e t}$ is faithful by (6), the map is injective. Suppose that

$$
\sigma: U V \longrightarrow \underline{\tilde{L F}}
$$

is a section of $\tilde{L F}$. Let $\left(u_{\alpha}: V_{\alpha} \hookrightarrow \underline{L F}\right)$ be an open covering family of $\underline{L F}$ such that for each $\alpha, L F \circ u_{\alpha}$ is an open embedding. Notice that in the following diagram, each square is a pullback in $\mathbb{L O C}$ :

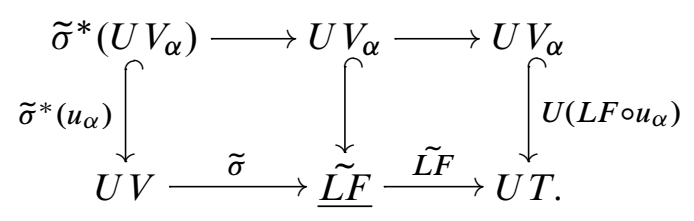

Observe that

$$
\left(\widetilde{\sigma}^{*}\left(U V_{\alpha}\right) \stackrel{\tilde{\sigma}^{*}\left(u_{\alpha}\right)}{\longrightarrow} U V\right)
$$

is an open cover covering. By (6), using $\psi_{T}$ we may assume without loss of generality that for each $\alpha$,

$$
\tilde{\sigma}^{*}\left(u_{\alpha}\right)=U\left(\sigma_{\alpha}: W_{\alpha} \hookrightarrow V\right),
$$

for some open covering

$$
\left(\sigma_{\alpha}: W_{\alpha} \hookrightarrow V\right)
$$


of $V$ in $S$. So we have an open covering $\left(\sigma_{\alpha}: W_{\alpha} \hookrightarrow V\right)$ such that for each $\alpha$, the composite

$$
U W_{\alpha} \longrightarrow U V \stackrel{\tilde{\sigma}}{\longrightarrow} \tilde{U}(\underline{L F})
$$

is the image under $U$ of an open embedding in $S$. Hence, by (5), there exists a $\sigma: V \rightarrow \underline{L F}$, such that $U(\sigma)=\tilde{\sigma}$.

Consequently, $F$ is isomorphic the sheaf of sections of $L F$. When viewing $F$ as a sheaf on $\mathcal{O}(T)$, we shall call $L F$ its étalé space in $S$. We can arrange this into a functor

$$
L: \operatorname{Sh}(T) \longrightarrow E t(T)
$$

The following theorem is immediate:

Theorem B.1 For every space $T$ in $S$, the étalé space functor $L$ is part of an adjoint equivalence

$$
\mathrm{Sh}(T) \stackrel{\Gamma}{\longleftarrow} E t(T),
$$

where $\Gamma$ associates a local homeomorphism $f: X \rightarrow T$ its sheaf of sections.

Proposition B.3 If $S$ is a category of spaces,

$$
U^{e t}: S^{e t} \longrightarrow \mathbb{L O C} \mathbb{C}^{e t}
$$

creates finite colimits.

Proof Let $F: J \rightarrow S^{e t}$ be any finite diagram. If $J$ is empty, we are done by (a), so suppose not. Suppose that $U^{e t} F$ has a colimit. Lets denote it by $X$ and lets denote the associated colimiting cocone by

$$
\rho: U^{e t} F \Longrightarrow \Delta_{X}
$$

This cocone corresponds canonically to a functor

$$
J \stackrel{\tilde{\rho}}{\longrightarrow} \mathbb{L} \mathbb{O} \mathbb{C}^{e t} / X
$$

Notice that the canonical map

$$
\coprod_{j \in J} \rho(j): \coprod_{j \in J} U F(j) \longrightarrow X
$$


is a surjective local homeomorphism. By (a),

$$
\coprod_{j \in J} U F(j) \cong U\left(\coprod_{j \in J} F(j)\right),
$$

so by (b), we can assume without loss of generality that $X=U(T)$ for some $T$. By (6), we can consider the composition

$$
J \stackrel{\tilde{\rho}}{\longrightarrow} \mathbb{L} \mathbb{O} \mathbb{C}^{e t} / X \stackrel{\psi_{T}}{\longrightarrow} S^{e t} / T,
$$

which corresponds to a cocone

$$
\bar{\rho}: F \longrightarrow \Delta_{T}
$$

for $F$ with vertex $T$. It readily follows that $U^{e t} \bar{\rho}$ is isomorphic to $\rho$ in the category of cocones for $U^{e t} F$, hence is colimiting, so we may assume without loss of generality that $\rho=U^{e t} \bar{\rho}$. It suffices to show that $\bar{\rho}$ is colimiting. Let $Z$ be an arbitrary space. Composition with $\bar{\rho}$ produces a canonical map

$$
\widehat{(\bar{\rho})}: \operatorname{Hom}_{S^{e t}}(T, Z) \longrightarrow \operatorname{Cocone}(F, Z) .
$$

We wish to show this map is a bijection. Notice that the following diagram commutes

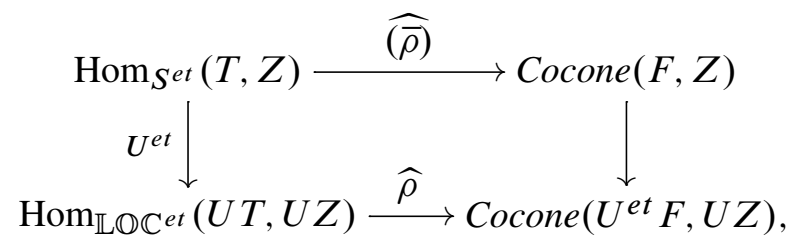

and the vertical arrows are injective since $U^{e t}$ is faithful by (6). It follows that $\widehat{(\bar{\rho})}$ is injective. It suffices to show that it is surjective. Let $\lambda: F \rightarrow \Delta_{Z}$ be a cocone on $Z$ for $F$. Then, since $\rho=U^{e t} \bar{\rho}$ is colimiting, there exists a unique $\theta: U T \rightarrow U Z$ such that

$$
U^{e t} \lambda=\left(U^{e t} F \stackrel{\rho}{\Longrightarrow} \Delta_{U} T \stackrel{\theta}{\Longrightarrow} \Delta_{U} Z\right) .
$$

Notice that $(F(j) \stackrel{\bar{\rho}(j)}{\longrightarrow} T)_{j}$ is a covering family of local homeomorphisms. Moreover, for each $j$, the composite

$$
U F(j) \stackrel{U \bar{\rho}(j)}{\longrightarrow} U T \stackrel{U \theta}{\longrightarrow} U Z
$$

is equal to $U(\lambda(j))$. By (5), it follows that there exists $\tilde{\theta}: Z \rightarrow T$ such that $U^{e t} \tilde{\theta}=\theta$. It follows that

$$
U^{e t} \widehat{(\bar{\rho})}=U^{e t} \lambda
$$


and since $U^{e t}$ is faithful, this implies

$$
\widehat{(\bar{\rho})}(\tilde{\theta})=\lambda .
$$

By the same proof, if $\bar{\rho}$ is any cocone for which $U^{e t} \bar{\rho}$ is colimiting, then $\bar{\rho}$ must be colimiting.

Remark If $S$ is a category of spaces and $S$ has, and $U$ preserves, arbitrary coproducts, by the same proof

$$
U^{e t}: S^{e t} \longrightarrow \mathbb{L O} \mathbb{C}^{e t}
$$

creates all colimits.

Corollary B.1 If $S$ is a category of spaces, the open cover topology on $S$ is subcanonical.

Proof It suffices to show that if $\left(T_{\alpha} \rightarrow T\right)$ is a covering family of local homeomorphisms, then

$$
T \cong \lim _{\longrightarrow}\left(\coprod T_{\alpha} \times_{T} T_{\beta} \rightrightarrows \coprod T_{\alpha}\right)
$$

By (3) and (4), this coequalizer may be computed in $S^{e t}$, which exists and is preserved by $U^{e t}$ by Proposition B.3. By the same proposition, it suffices to show that

$$
U T \cong \lim _{\longrightarrow} U\left(\coprod T_{\alpha} \times_{T} T_{\beta} \rightrightarrows \coprod T_{\alpha}\right)
$$

Notice that

$$
\stackrel{\lim }{\longrightarrow}\left(\coprod T_{\alpha} \times_{T} T_{\beta} \rightrightarrows \coprod T_{\alpha}\right) \cong \underset{\lim }{\longrightarrow}\left(\coprod U T_{\alpha} \times_{U T} U T_{\beta} \rightrightarrows \coprod U T_{\alpha}\right)
$$

Since the open cover topology on $\mathbb{L O C}$ is subcanonical, we are done.

\section{Appendix C: Sheaves in groupoids versus stacks}

Definition C.1 Let $\mathcal{C}$ be a small category. A strict presheaf in groupoids over $\mathcal{C}$ is a strict 2-functor $F: \mathcal{C}^{o p} \rightarrow G p d$ to the 2-category of (small) groupoids. Notice that this is the same as a $1-$ functor $\mathcal{C}^{o p} \rightarrow \tau_{1}(G p d)$, where the target is the 1-category of groupoids. A morphism of strict presheaves is a strict natural transformation (i.e., a natural transformation between their corresponding 1-functors into $\tau_{1}(G p d)$ ). A 2morphism between two natural transformations $\alpha_{i}: F \Rightarrow G, i=1,2$, is an assignment to each object $C$ of $\mathcal{C}$ a natural transformation

$$
w(C): \alpha_{1}(C) \Longrightarrow \alpha_{2}(C),
$$

subject to the following condition: 
For all $f: D \rightarrow C$, we have two functors from $F(C)$ to $G(D)$, namely

$$
G(f) \alpha_{1}(C)=\alpha_{1}(D) F(f) \quad \text { and } \quad G(f) \alpha_{2}(C)=\alpha_{2}(D) F(f) .
$$

Given our assignment $C \mapsto w(C)$, we have two different natural transformations between these functors: $G(f) w(C)$ and $w(D) F(f) . w$ is called a modification if these two natural transformations are equal. Modifications are the 2-cells of strict presheaves. This yields a strict 2-category of strict presheaves in groupoids $P \operatorname{sh}(\mathcal{C}, G p d)$.

Proposition C.1 The 2-category $\operatorname{Psh}(\mathcal{C}, G p d)$ is equivalent to the 2-category of groupoid objects in $\mathrm{Set}^{{ }^{c o p}}$.

Proof Let $(\cdot)_{i}: \tau_{1}(G p d) \rightarrow$ Set, $i=0,1,2$ be the functors which associate to a groupoid $\mathcal{G}$ its set of objects $\mathcal{G}_{0}$, its set of arrows $\mathcal{G}_{1}$ and its set $\mathcal{G}_{2}$ of composable arrows respectively. Let $F: \mathcal{C}^{o p} \rightarrow \tau_{1}(G p d)$ be a strict presheaf of groupoids. Then each $F_{i}$ is an ordinary presheaf of sets. Moreover, for each $C, F(C)$ is a groupoid, which we may write as demanding certain diagram involving each $F(C)_{i}$ to commute. These assemble to a corresponding diagram for the global $F_{i}$, showing they form a groupoid object in $\operatorname{Set}^{c o p}, Q(F)$. Given 1-morphism $\alpha: F \Rightarrow G$ in $P \operatorname{sh}(\mathcal{C}, G p d)$, let $Q(\alpha): Q(F) \rightarrow Q(F)$ be the internal functor with components $Q(\alpha)_{i}(C)=\alpha(C)_{i}$ for $i=0,1$. Finally, let $w$ be a modification from $\alpha$ to $\beta$. Then, in particular, for each $C, w(C): \alpha(C) \Rightarrow \beta(C)$ is a natural transformation, so it is a map $w(C): F(C)_{0} \rightarrow G(C)_{1}$ satisfying the obvious properties. It is easy to check that the conditions for $w$ to be a modification are precisely those for the family $\left(w(C): F(C)_{0} \rightarrow G(D)_{1}\right)$ to assemble into a natural transformation

$$
Q(w): F_{0} \Longrightarrow G_{1} \text {. }
$$

Since $w$ is point-wise a natural transformation, $Q(w)$ is an internal natural transformation. It is easy to check that this is indeed an equivalence of 2-categories with an explicit inverse on objects given by

$$
\mathbb{G} \longmapsto \operatorname{Hom}(\cdot, \mathbb{G}) .
$$

Definition C.2 Let $(\mathcal{C}, J)$ be a Grothendieck site. Then a sheaf of groupoids is a strict presheaf $F: \mathcal{C}^{o p} \rightarrow \tau_{1}(G p d)$ such that for any covering family $\left(C_{i} \rightarrow C\right)_{i}$, the induced morphism

$$
F(C) \longrightarrow \underset{\leftarrow}{\lim }\left[\prod_{i} F\left(C_{i}\right) \rightrightarrows \prod_{i, j} F\left(C_{i j}\right)\right]
$$

is an isomorphism of groupoids. Sheaves of groupoids form a full sub-2-category $\operatorname{Sh}(\mathcal{C}, G p d)$ of strict presheaves of groupoids. 
The following proposition is easily checked:

Proposition C.2 The $2-$ functor $Q: P s h(\mathcal{C}, G p d) \rightarrow G p d\left(\mathrm{Set}^{\mathcal{C}^{o p}}\right)$ restricts to an equivalence $Q: \operatorname{Sh}(\mathcal{C}, G p d) \rightarrow G p d(\operatorname{Sh}(\mathcal{C}))$.

Analogously to sheaves of sets, there is a 2-adjunction

$$
\operatorname{Sh}(\mathcal{C}, G p d) \underset{i}{\stackrel{s h}{\leftrightarrows}} \operatorname{Psh}(\mathcal{C}, G p d)
$$

where $s h$ denotes sheafification.

Denote by $j: P \operatorname{sh}(\mathcal{C}, G p d) \rightarrow G p d^{\mathcal{C}^{o p}}$ the "inclusion" of strict presheaves into weak presheaves. We use quotations since this functor is not full. The following proposition is standard:

Proposition C.3 Let $\mathcal{Z}$ be a strict presheaf of groupoids. Then

$$
a \circ j(\mathcal{Z}) \simeq a \circ j \circ i \circ \operatorname{sh}(\mathcal{Z}),
$$

where $a$ denotes stackification.

In other words, if you start with a strict presheaf of groupoids, sheafify it to a sheaf of groupoids and then stackify the result, this is equivalent to stackifying the original presheaf.

Corollary C.1 Every stack is equivalent to $a \circ j \circ i(\mathcal{W})$ for some sheaf of groupoids $\mathcal{W}$.

\section{Appendix D: Proof of Theorem 3.2}

In this appendix, we will prove that the generalized action groupoid construction described in Section 3.1 yields a concrete description of étalé realization. For technical reasons, we start by fixing an ambient Grothendieck universe $\mathcal{U}$. Recall the following definition from [1, Exposé ii]:

Definition D.1 A locally $\mathcal{U}$-small Grothendieck site $(\mathscr{E}, V)$ is called a $\mathcal{U}$-site if there exists a $\mathcal{U}$-small set of objects $G$, called topological generators, such that every object $E \in \mathscr{E}$ admits $V$-cover by a family of morphisms all of whose sources are in $G$.

Theorem D.1 [1, Exposé ii, Théorème 3.4] If $(\mathscr{E}, V)$ is a $\mathcal{U}$-site, then the category of presheaves of $\mathcal{U}$-small sets on $\mathcal{E}$ is locally $\mathcal{U}$-small. Moreover, the full subcategory thereof consisting of $\mathcal{U}$-small $V$-sheaves is reflective, and the reflector is left exact, hence this subcategory is a $\mathcal{U}$-topos. 
Remark In particular, a $\mathcal{U}$-site is not necessarily $\mathcal{U}$-small. An important example of a $\mathcal{U}$-site which is not $\mathcal{U}$-small is the following:

Suppose $(\mathcal{C}, J)$ is a $\mathcal{U}$-small site. Let $\mathcal{E}:=\operatorname{Sh}_{J}^{\mathcal{U}}(\mathcal{C})$ be the $\mathcal{U}$-topos of $J$-sheaves of $\mathcal{U}$-small sets. Equip $\mathcal{E}$ with the canonical topology, which is generated by jointly surjective epimorphic families. Denote this site by $(\mathcal{E}$, can $)$. This site is clearly not $\mathcal{U}$-small, but it is a $\mathcal{U}$-site, since the set of representable sheaves is $\mathcal{U}$-small (since it is a copy of $\mathcal{C}$ ) and topologically generates $\mathcal{E}$ (because of the Yoneda lemma). Moreover, the category of $\mathcal{U}$-small sheaves on this site, is canonically equivalent to $\mathcal{E}$ itself. More generally, if $(\mathcal{C}, J)$ is not $\mathcal{U}$-small, but just a $\mathcal{U}$-site, $(\mathcal{E}$, can $)$ is still a $\mathcal{U}$-site; its topological generators are the image of those of $\mathcal{C}$ under the Yoneda embedding.

Lemma D.2 Let $\mathcal{E}$ be a $\mathcal{U}$-small topos, $(\mathcal{D}, K)$ be a $\mathcal{U}$-small site and

$$
G: \mathcal{E} \longrightarrow \mathrm{St}_{K}^{\mathcal{U}}(\mathcal{D})
$$

be a left exact 2 -functor from $\mathcal{E}$ into the 2 -topos (in $\mathcal{U}$ ) of $K$-stacks of (essentially) $\mathcal{U}$-small groupoids, which preserves coproducts and epimorphisms. Denote by

$$
\hat{y}_{\mathcal{E}}: \mathcal{E} \longrightarrow \operatorname{St}_{\text {can }}^{\mathcal{U}}(\mathcal{E})
$$

the Yoneda embedding of $\mathcal{E}$ into the 2-topos of $\mathcal{U}$-small stacks on $\mathcal{E}$. Then there exists (an essentially unique) $\mathcal{U}$-small weak colimit preserving 2 -functor

$$
L_{\mathcal{E}}^{\mathcal{U}}: \operatorname{St}_{c a n}^{\mathcal{U}}(\mathcal{E}) \longrightarrow \operatorname{St}_{K}^{\mathcal{U}}(\mathcal{D})
$$

which when restricted to $\mathcal{E}$ along $y_{\mathcal{E}}$ agrees with $G$ (up to equivalence).

Proof Let $\mathcal{V}$ be a larger Grothendieck universe such that $\mathcal{U} \in \mathcal{V}$. Then $(\mathcal{E}$, can $)$ is a $\mathcal{V}$-small site. Consider the canonical inclusion

$$
i_{\mathcal{D}}^{\mathcal{V}}: \operatorname{St}_{K}^{\mathcal{U}}(\mathcal{D}) \longrightarrow \mathrm{St}_{K}^{\mathcal{V}}(\mathcal{D}) .
$$

By [13, Remark 6.3.5.17], this inclusion preserves $\mathcal{U}$-small weak colimits (apply $\tau_{\leq 1}$ to functor in this proposition, and note that $\tau_{\leq 1}$ is a colimit preserving functor of infinity categories). Let $\widehat{\mathcal{E}}$ denote the 2-topos in $\mathcal{V}$ of $\mathcal{V}$-small weak presheaves of groupoids on $\mathcal{E}$. Let $L_{\mathcal{E}}^{\mathcal{V}}$ denote the weak left Kan extension of $i_{\mathcal{D}}^{\mathcal{V}} \circ G$ along the Yoneda embedding $\hat{y}^{\mathcal{V}}$ of $\mathcal{E}$ into $\widehat{\mathcal{E}}$. Denote its right adjoint by $R_{\mathcal{E}}^{\mathcal{V}}$. Explicitly, for $\mathcal{Z} \in \mathrm{St}_{K}^{\mathcal{V}}(\mathcal{D}), R_{\mathcal{E}}^{\mathcal{V}}(\mathcal{Z})$ is the weak presheaf that assigns $E \in \mathcal{E}$, the groupoid

$$
\begin{aligned}
R_{\mathcal{E}}^{\mathcal{V}}(\mathcal{Z})(E) & \simeq \operatorname{Hom}\left(L_{\mathcal{E}}^{\mathcal{V}} \hat{y}^{\mathcal{V}}(E), \mathcal{Z}\right) \\
& \simeq \operatorname{Hom}\left(i_{\mathcal{D}}^{\mathcal{V}} G(E), \mathcal{Z}\right) .
\end{aligned}
$$


Since $G$ and $i_{\mathcal{D}}^{\mathcal{V}}$ both preserve coproducts and epimorphisms, and are left exact (the latter since limits are computed point-wise) their composite preserves covers, and since $\mathcal{Z}$ is a stack, it follows that this presheaf satisfies descent for the canonical topology on $\mathcal{E}$, so is an object of $\mathrm{St}_{\text {can }}^{\mathcal{V}}(\mathcal{E})$. Hence, by abuse of notation, there is an induced 2-adjunction

$$
\operatorname{St}_{\text {can }}^{\mathcal{V}}(\mathcal{E}) \underset{L_{\mathcal{E}}^{\mathcal{V}}}{\stackrel{R_{\mathcal{E}}^{\mathcal{V}}}{\leftrightarrows}} \operatorname{St}_{K}^{\mathcal{V}}(\mathcal{D})
$$

The 2 -functor $L_{\mathcal{E}}^{\mathcal{V}}$ is uniquely determined up to equivalence by the fact that it is $\mathcal{V}$-small weak colimit preserving, and agrees up to equivalence with $i_{\mathcal{D}}^{\mathcal{V}} \circ G$ when restricted to $\mathcal{E}$ along $\hat{y}^{\mathcal{V}}$. Define

$$
L_{\mathcal{E}}^{\mathcal{U}}:=L_{\mathcal{E}}^{\mathcal{V}} \circ i_{\mathcal{E}}^{\mathcal{V}}
$$

where $i_{\mathcal{E}}^{\mathcal{V}}$ is the canonical inclusion

$$
i_{\mathcal{E}}^{\mathcal{V}}: \operatorname{St}_{\text {can }}^{\mathcal{U}}(\mathcal{E}) \longrightarrow \mathrm{St}_{\text {can }}^{\mathcal{V}}(\mathcal{E})
$$

which is $\mathcal{U}$-small weak colimit preserving. It follows that $L_{\mathcal{E}}^{\mathcal{U}}$ is $\mathcal{U}$-small weak colimit preserving, and by construction, when restricted to $\mathcal{E}$ along the Yoneda embedding $\hat{y}^{\mathcal{U}}$ of $\mathcal{E}$ into $\mathcal{U}$-small stacks over $\mathcal{E}$, it agrees up to equivalence with $G$. It suffices to show that the essential image of $L_{\mathcal{E}}^{\mathcal{U}}$ lies in the essential image of $i_{\mathcal{D}}^{\mathcal{V}}$, i.e., $\mathcal{U}$-small $K$-stacks. Let $\mathcal{Y} \in \operatorname{St}_{\text {can }}^{\mathcal{H}}(\mathcal{E})$. Then $\mathcal{Y}$ is a stack over the topos $\mathcal{E}$ in the sense of [6], hence there exists groupoid object $\mathcal{K} \in \operatorname{Gpd}(\mathcal{E})$ such that $\mathcal{Y} \simeq[\mathcal{K}]$ is its stack completion. In particular, this implies that $\mathcal{Y}$ is the weak colimit of the truncated semisimplicial diagram

$$
\mathcal{K}_{2} \rightrightarrows \mathcal{K}_{1} \rightrightarrows \mathcal{K}_{0}
$$

when viewed as a diagram in $\operatorname{St}_{\text {can }}^{\mathcal{U}}(\mathcal{E})$, i.e., taking the weak colimit after applying $\hat{y}^{\mathcal{U}}$, the Yoneda embedding into $\mathcal{U}$-small stacks over $\mathcal{E}$. Hence

$$
\begin{aligned}
L_{\mathcal{E}}^{\mathcal{U}}(\mathcal{Y}) & =L_{\mathcal{E}}^{\mathcal{V}} i_{\mathcal{E}}^{\mathcal{V}}(\mathcal{Y}) \\
& \simeq L_{\mathcal{E}}^{\mathcal{V}} i_{\mathcal{E}}^{\mathcal{V}}\left(\underline{\operatorname{holim}} \hat{y}^{\mathcal{U}} \mathcal{K}_{n}\right) \\
& \simeq \stackrel{\operatorname{holim}}{\longrightarrow} L_{\mathcal{E}}^{\mathcal{V}} \hat{y}^{\mathcal{V}} \mathcal{K}_{n} \\
& \simeq \stackrel{\operatorname{holim}}{\longrightarrow} i_{\mathcal{D}}^{\mathcal{V}} G\left(\mathcal{K}_{n}\right) \\
& \simeq i_{\mathcal{D}}^{\mathcal{V}}\left(\stackrel{\operatorname{holim}}{\longrightarrow} G\left(\mathcal{K}_{n}\right)\right) .
\end{aligned}
$$

Therefore, the essential image of $L_{\mathcal{E}}^{\mathcal{U}}$ consists entirely of $\mathcal{U}$-small $K$-stacks. 
Let $\mathcal{X} \simeq[\mathcal{H}]$ be an étale stack, with $\mathcal{H}$ an étale $S$-groupoid. Consider the 2 -functor

$$
\mathcal{H} \ltimes: G p d(\mathcal{B H}) \longrightarrow\left(S^{e t}-G p d\right) / \mathcal{H}
$$

from Section 3.1. Denote by $G$ the composition

$$
\mathcal{B H} \stackrel{q}{\longrightarrow} \operatorname{Gpd}(\mathcal{B H}) \stackrel{\mathcal{H} \ltimes}{\longrightarrow}\left(S^{e t}-G p d\right) / \mathcal{H} \stackrel{Y}{\longrightarrow} \operatorname{St}(S / X),
$$

where $q$ is the canonical inclusion.

Proposition D.1 The 2-functor $G$ as defined above preserves coproducts and epimorphisms.

Proof The fact that $G$ preserves coproducts can be checked immediately. As far as epimorphisms, suppose that

$$
\varphi: E \longrightarrow F
$$

is an epimorphism in $\mathcal{B H}$. To show that $G(\varphi)$ is an epimorphism in $\operatorname{St}(S / X)$, it suffices to show that the map

$$
t \circ p r_{1}:(\mathcal{H} \ltimes F)_{1} \times_{F} E \longrightarrow F
$$

is an epimorphism in $\mathcal{B H}$, where

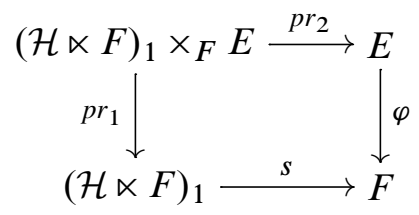

is the pullback diagram in $\mathcal{B H}$. However, $(\mathcal{H} \ltimes F)_{1}$ is itself the pullback

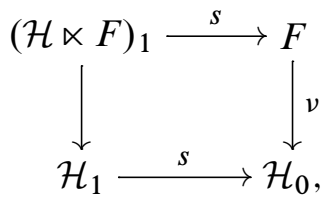

where $v$ is the moment map of $F$. Since $\varphi$ is, in particular, a map in $S / \mathcal{H}_{0}, v \circ \varphi=\mu$, where $\mu$ is the moment map of $E$. Hence $(\mathcal{H} \ltimes F)_{1} \times{ }_{F} E$ is in fact the fibered product

$$
\mathcal{H}_{1} \times \mathcal{H}_{0} E=(\mathcal{H} \ltimes E)_{1} .
$$

The map $t \circ p r_{1}$ can then be identified with $t \circ \mathcal{H} \ltimes(\varphi)$ which is an epimorphism since $t$ is and $\mathcal{H} \ltimes(\varphi)$ is the pullback of $\varphi$ along

$$
s:(\mathcal{H} \ltimes F)_{1} \longrightarrow F .
$$


Lemma D.3 The composite

$$
Y \circ \mathcal{H} \ltimes: \operatorname{Gpd}(\mathcal{B H}) \longrightarrow \operatorname{St}(S / \mathcal{X})
$$

preserves epimorphisms and weak pullbacks.

Proof Suppose that $\varphi: \mathcal{K} \rightarrow \mathcal{L}$ is an epimorphism in $\operatorname{Gpd}(\mathcal{B H})$. This implies that the induced map

$$
t \circ p r_{1}: \mathcal{L}_{1} \times \mathcal{L}_{0} \mathcal{K}_{0} \longrightarrow \mathcal{L}_{0}
$$

is an epimorphism. In particular, this means that, when viewed as a map of underlying spaces, it is a surjective local homeomorphism, i.e., an étale cover. There is a canonical map

$$
\mathcal{L}_{1} \times \mathcal{L}_{0} \mathcal{K}_{0} \longrightarrow(\mathcal{H} \ltimes \mathcal{L})_{1} \times \mathcal{L}_{0} \mathcal{K}_{0}
$$

induced by the canonical homomorphism $\tau_{\mathcal{L}}: \mathcal{L} \rightarrow(\mathcal{H} \ltimes \mathcal{L}$ ) (see the remark directly preceding Proposition 3.1) such that the following diagram commutes:

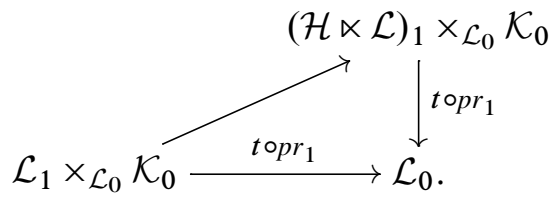

Hence

$$
t \circ p r_{1}:(\mathcal{H} \ltimes \mathcal{L})_{1} \times \mathcal{L}_{0} \mathcal{K}_{0} \longrightarrow \mathcal{L}_{0}
$$

is a surjective local homeomorphism. This implies that $Y \circ \mathcal{H} \ltimes(\varphi)$ is an epimorphism. To show that $Y \circ \mathcal{H} \ltimes$ preserves weak pullbacks, it suffices to show that $\mathcal{H} \ltimes$ does, since $Y$ preserves finite weak limits. Suppose that

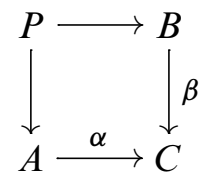

is a weak pullback diagram in $\mathcal{B H}$. Explicitly, we may describe $P$ by its objects being triples

$$
(a, b, l) \in A_{0} \times B_{0} \times C_{1}
$$

such that

$$
l: \alpha(a) \longrightarrow \beta(b)
$$


with the obvious structure of an étale $\mathcal{H}$-space (we can take the moment map $\xi_{0}$ to be the projection onto $B_{0}$ followed by its moment map $v_{0}$ ). Its arrows from

$$
(a, b, l) \longrightarrow\left(a^{\prime}, b^{\prime}, l^{\prime}\right)
$$

can be described by pairs

$$
\left(k_{a}, k_{b}\right) \in A_{1} \times B_{1}
$$

such that the following diagram commutes:

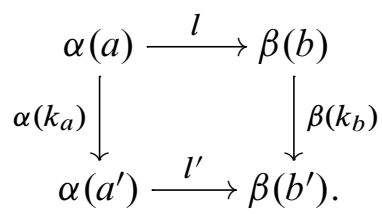

This condition on the arrows can be expressed as a pullback diagram, hence they also inherits the structure of an étale $\mathcal{H}$-space. Now, the objects of $\mathcal{H} \ltimes P$ are the same as $P$. The arrows

$$
(a, b, l) \longrightarrow\left(a^{\prime}, b^{\prime}, l^{\prime}\right)
$$

in $\mathcal{H} \ltimes P$ can be described by triples $\left(h, k_{h a}, k_{h b}\right)$ such that

$$
\left(k_{h a}, k_{h b}\right):(h a, h b, h l) \longrightarrow\left(a^{\prime}, b^{\prime}, l^{\prime}\right)
$$

is an arrow in $P$. These of course assemble into a space which can be constructed via pullbacks as well, with moment map $\xi_{1}$. With the choice of moment maps $\xi, \mathcal{H} \ltimes P$ becomes an $S$-groupoid over $\mathcal{H}$ by factoring it through

$$
\theta_{B}: \mathcal{H} \ltimes B \longrightarrow \mathcal{H} .
$$

Let $P^{\prime}$ denote the weak pullback

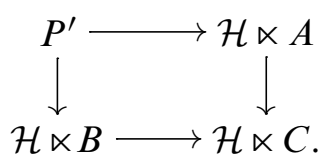

Its objects can be described by quadruples

$$
(a, b, h, l) \in A_{0} \times B_{0} \times \mathcal{H}_{1} \times C_{1}
$$

such that

$$
k: h \alpha(a) \longrightarrow \beta(b) .
$$

A quick calculation shows that its arrows

$$
(a, b, h, l) \longrightarrow\left(a^{\prime}, b^{\prime}, h^{\prime}, l^{\prime}\right)
$$


can be described by quadruples

$$
\left(h_{a}, k_{a}, h_{b}, k_{b}\right) \in \mathcal{H}_{1} \times A_{1} \times \mathcal{H}_{1} \times B_{1}
$$

such that

$$
k_{a}: h_{a} a \longrightarrow a^{\prime} \text { and } k_{b}: h_{b} b \longrightarrow b^{\prime}
$$

and such that

$$
\left(h^{\prime} h_{a}, l^{\prime} \circ\left(h^{\prime} \cdot \alpha\left(k_{a}\right)\right)\right)=\left(h_{b} h, \beta\left(k_{b}\right) \circ\left(h_{b} \cdot l\right)\right) .
$$

We may regard $P^{\prime}$ as an $S$-groupoid over $\mathcal{H}$ by factoring it through its canonical projection onto $\mathcal{H} \ltimes B$.

There is of course a canonically induced map

$$
F: \mathcal{H} \ltimes P \longrightarrow P^{\prime}
$$

coming from the cone obtained by applying $\mathcal{H} \ltimes$ to the diagram expressing $P$ as a pullback. On objects, $F$ sends a triple

$$
(a, b, l) \longmapsto\left(a, b, \mathbb{1}_{\mu_{1}(l)}, l\right),
$$

where

$$
\mu_{1}: C_{1} \longrightarrow \mathcal{H}_{0}
$$

is the moment map. On arrows it sends

$$
\left(h, k_{h a}, k_{h b}\right) \longmapsto\left(h, k_{h a}, h, k_{h b}\right) .
$$

Define a homomorphism $\Lambda: P^{\prime} \rightarrow P$ on objects by

$$
(a, b, h, l) \longmapsto\left(h a, b, \mathbb{1}_{\mu_{1}(l)}, l\right)
$$

and on arrows by sending quadruples

$$
\left(h_{a}, k_{a}, h_{b}, k_{b}\right):(a, b, h, l) \longrightarrow\left(a^{\prime}, b^{\prime}, h^{\prime}, l^{\prime}\right)
$$

to triples

$$
\left(h^{\prime} h_{a} h^{-1}=h_{b}, h^{\prime} \cdot k_{a}, k_{b}\right) .
$$

Notice that $\Lambda$ strictly commutes over $\mathcal{H}$ and $\Lambda \circ F=\operatorname{id}_{\mathcal{H} \ltimes P}$. Moreover, consider the continuous map

$$
\omega: P_{0}^{\prime} \longrightarrow P_{1}^{\prime}
$$

given by

$$
(a, b, h, l) \longmapsto\left(h, \mathbb{1}_{h a}, \mathbb{1}_{v_{0}(b)}, \mathbb{1}_{b}\right),
$$


where $v_{0}$ is the moment map of $B$. Notice that

$$
\omega(a, b, h, l):(a, b, h, l) \longrightarrow\left(h a, b, \mathbb{1}_{\mu_{1}(l)}, l\right) .
$$

It follows that $\omega$ is an internal natural transformation

$$
\omega: \operatorname{id}_{P}^{\prime} \Longrightarrow F \circ \Lambda \text {. }
$$

It is easy to check that it is indeed a $2-$ morphism in $S-G p d / \mathcal{H}$, hence $F$ is an equivalence.

Corollary D.1 There exists a weak colimit preserving 2-functor

$$
L_{\mathcal{B H}}: \operatorname{St}_{\text {can }}(\mathcal{B H}) \longrightarrow \operatorname{St}(S / \mathcal{X}),
$$

whose restriction to $\mathcal{B H}$ along the Yoneda embedding

$$
\hat{y}_{\mathcal{B H}}: \mathcal{B H} \hookrightarrow \operatorname{St}_{\text {can }}(\mathcal{B H})
$$

agrees with $G$ from (6) up to equivalence.

(We have suppressed the role of the Grothendieck universe $\mathcal{U}$ for simplicity.)

Proof The 2-functor $G$ can be easily checked to preserve the terminal object, so by Lemma D.3, it follows that it is left exact. Combining this with Proposition D.1, the result then follows from Lemma D.2.

\section{Lemma D.4 Let}

$$
Y:\left(S^{e t}-G p d\right) / \mathcal{H} \longrightarrow \operatorname{St}(S) / \mathcal{X}
$$

be the 2 -functor which sends a groupoid $\varphi: \mathcal{G} \rightarrow \mathcal{H}$ over $\mathcal{H}$ to

$$
[\varphi]:[\mathcal{G}] \longrightarrow[\mathcal{H}]=\mathcal{X} .
$$

Then for $U \subset \mathcal{H}_{0}$ an open subset, the stacks $y\left(U \hookrightarrow \mathcal{H}_{0} \rightarrow \mathcal{X}\right)$ and

$$
Y\left(\theta_{m_{U}}\right)=\left[\theta_{m_{U}}\right]
$$

are canonically equivalent in $\operatorname{St}(S) / \mathcal{X}$, where $m_{U}$ is the equivariant sheaf associated to the representable $U \in \operatorname{Site}(\mathcal{H})_{0}$ (Definition 2.26), and

$$
\theta_{m_{U}}: \mathcal{H} \ltimes m_{U} \longrightarrow \mathcal{H}
$$

is as in the remark directly preceding Proposition 3.1. 
Proof $\mathcal{H} \ltimes m_{U}$ has objects $s^{-1}(U)$ and arrows are of the form

$$
(h, \gamma): \gamma \longrightarrow h \circ \gamma \text {. }
$$

Define an internal functor

$$
f_{U}: \mathcal{H} \ltimes m_{U} \longrightarrow U^{\mathrm{id}}
$$

on objects as

$$
s^{-1}(U) \stackrel{s}{\longrightarrow} U
$$

and on arrows by

$$
(h, \gamma) \longmapsto s(\gamma)
$$

Define another internal functor

$$
g_{U}: U^{\mathrm{id}} \longrightarrow \mathcal{H} \ltimes m_{U}
$$

on objects as

$$
x \longmapsto \mathbb{1}_{x}
$$

and on arrows as

$$
x \longmapsto\left(\mathbb{1}_{x}, \mathbb{1}_{x}\right)
$$

Clearly

$$
f_{U} \circ g_{U}=\mathrm{id}_{U^{\text {id }}} .
$$

Moreover, there is a canonical internal natural transformation

$$
\lambda_{U}: g_{U} \circ f_{U} \Longrightarrow \mathrm{id}_{\mathcal{H} \ltimes m_{U}},
$$

given by

$$
\lambda_{U}(\gamma)=\left(\gamma, \mathbb{1}_{s(\gamma)}\right)
$$

Denote by $a_{U}: U^{\text {id }} \rightarrow \mathcal{H}$, the composite

$$
U \hookrightarrow \mathcal{H}_{0} \longrightarrow \mathcal{H} .
$$

Notice that $g_{U}$ extends to a morphism from $a_{U}: U^{\text {id }} \rightarrow \mathcal{H}$ to $\mathcal{H} \ltimes m_{U}$ in $\left(S^{e t}-G p d\right) / \mathcal{H}$ as $\theta_{m_{U}} \circ g_{U}=a_{U}$. Note that the formula

$$
\alpha_{U}(\gamma)=\gamma^{-1}
$$

defines an internal natural transformation

$$
\alpha_{U}: \theta_{m_{U}} \Longrightarrow a_{U} \circ f_{U} .
$$


Hence $\left(f_{U}, \alpha_{U}\right)$ is morphism in $\left(S^{e t}-G p d\right) / \mathcal{H}$ from $\theta_{m_{U}}$ to $a_{U}$. It is easy to check that $\lambda_{U}$ is in fact a $2-$ cell in $\left(S^{e t}-G p d\right) / \mathcal{H}$. Hence $a_{U}$ and $\theta_{m_{U}}$ are canonically equivalent, so the same is true of their images under $Y$.

Consider the functor

$$
m: \operatorname{Site}(\mathcal{H}) \longrightarrow \mathcal{B H}
$$

from Proposition 2.6. Then, this is a morphism of sites, and in light of the aforementioned proposition, it induces an equivalence of bicategories

$$
m_{!}: \operatorname{St}(\operatorname{Site}(\mathcal{H})) \longrightarrow \operatorname{St}_{c a n}(\mathcal{B H}) \text {. }
$$

The 2-functor $m$ ! is the weak left Kan extension of $\hat{y}_{\mathcal{B H}} \circ m$ (See Corollary D.1 for the notation) along the Yoneda embedding

$$
\hat{y}_{\mathcal{H}}: \operatorname{Site}(\mathcal{H}) \hookrightarrow \operatorname{St}(\operatorname{Site}(\mathcal{H})) .
$$

In other words, it is the unique weak colimit preserving 2-functor whose restriction to $\operatorname{Site}(\mathcal{H})$ along $\hat{y}_{\mathcal{H}}$ agrees with $\hat{y}_{\mathcal{B} \mathcal{H}} \circ m$ up to equivalence.

Corollary D.2 The étale realization 2-functor

$$
\bar{L}: \operatorname{St}(\operatorname{Site}(\mathcal{H})) \longrightarrow \operatorname{St}(S / \mathcal{X})
$$

from Corollary 2.4 is equivalent to $L_{\mathcal{B H}} \circ m_{!}$.

Proof This follows from Lemma D.4 together with Corollary D.1.

Theorem D.5 Consider the canonical 2-functor

$$
[\cdot]_{\mathcal{B H}}: \operatorname{Gpd}(\mathcal{B H}) \longrightarrow \operatorname{St}(\operatorname{Site}(\mathcal{H})),
$$

which associates a groupoid object $\mathcal{K}$ in $\mathcal{B H}$ with its stack completion. Then $[\cdot]_{\mathcal{B H}}$ is essentially surjective and faithful (but not in general full), and the 2 -functors $\bar{L} \circ[\cdot]_{\mathcal{B H}}$ and $Y \circ \mathcal{H} \ltimes$ are equivalent.

Proof The fact that $[\cdot]_{\mathcal{B H}}$ is essentially surjective follows from the fact that every stack is equivalent to a strict 2 -functor, which is in particular a sheaf of groupoids, i.e., a groupoid object in sheaves. The fact that is faithful follows from the fact that sheaves of groupoids considered as weak presheaves are separated (i.e., prestacks). Let $\mathcal{K}$ be a 
groupoid object in $\mathcal{B H}$. Then the following is a $2-$ Cartesian cube all of whose edges are epimorphisms:

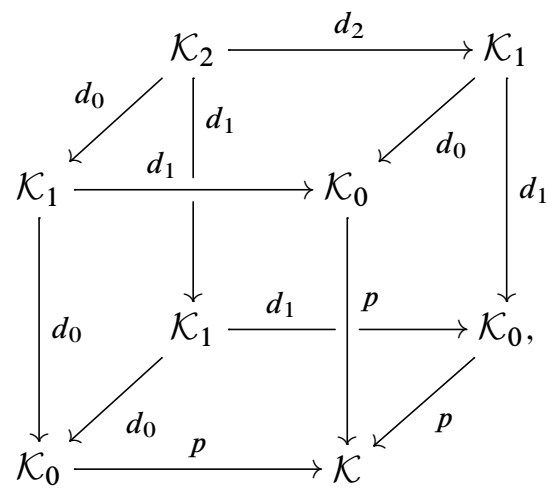

where $p: \mathcal{K}_{0} \rightarrow \mathcal{K}$ is the canonical map. From Lemma D.3,

$$
Y \circ \mathcal{H} \ltimes(p):\left[\mathcal{H} \ltimes \mathcal{K}_{0}\right] \longrightarrow[\mathcal{H} \ltimes \mathcal{K}]
$$

is an epimorphism, and also, applying $Y \circ \mathcal{H} \ltimes$ to the above cube, results in another 2-Cartesian cube, this time in the 2-topos $\operatorname{St}(S / \mathcal{X})$, all of whose edges are again epimorphisms. The fact that this cube is Cartesian means that the diagram obtained by deleting the vertex $[\mathcal{H} \ltimes \mathcal{K}]$ and all edges into it, namely

$$
\left[\mathcal{H} \ltimes \mathcal{K}_{2}\right] \rightrightarrows\left[\mathcal{H} \ltimes \mathcal{K}_{1}\right] \rightrightarrows\left[\mathcal{H} \ltimes \mathcal{K}_{0}\right]
$$

is the truncated semisimplicial Cěch nerve of the epimorphism $Y \circ \mathcal{H} \ltimes(p)$. From [13], since $\operatorname{St}(S / \mathcal{X})$ is a 2-topos, this implies that

$$
[\mathcal{H} \ltimes \mathcal{K}] \simeq \underline{\operatorname{holim}}\left(\left[\mathcal{H} \ltimes \mathcal{K}_{2}\right] \rightrightarrows\left[\mathcal{H} \ltimes \mathcal{K}_{1}\right] \rightrightarrows\left[\mathcal{H} \ltimes \mathcal{K}_{0}\right]\right)
$$

Notice that in the 2-topos $\operatorname{St}(\operatorname{Site}(\mathcal{H})$,

$$
[\mathcal{K}]_{\mathcal{B H}} \simeq \underset{\operatorname{holim}}{\longrightarrow}\left(\mathcal{K}_{2} \rightrightarrows \mathcal{K}_{1} \rightrightarrows \mathcal{K}_{0}\right)
$$

From Corollary D.2, this implies that

$$
\bar{L}[\mathcal{K}]_{\mathcal{B H}} \simeq L_{\mathcal{B H}} \circ m_{!}\left([\mathcal{K}]_{\mathcal{B H}}\right) \simeq \underline{\operatorname{holim}}\left(\left[\mathcal{H} \ltimes \mathcal{K}_{2}\right] \rightrightarrows\left[\mathcal{H} \ltimes \mathcal{K}_{1}\right] \rightrightarrows\left[\mathcal{H} \ltimes \mathcal{K}_{0}\right]\right)
$$

Hence

$$
\bar{L}[\mathcal{K}]_{\mathcal{B H}} \simeq[\mathcal{H} \ltimes \mathcal{K}] .
$$

We leave the rest of the details to the reader. 


\section{References}

[1] M Artin, A Grothendieck, J L Verdier, editors, Théorie des topos et cohomologie étale des schémas, Tome 1: Théorie des topos (SGA IV), Lecture Notes in Math. 269, Springer, Berlin (1972) MR0354652

[2] M Bunge, An application of descent to a classification theorem for toposes, Math. Proc. Cambridge Philos. Soc. 107 (1990) 59-79 MR1021873

[3] C Butz, I Moerdijk, Representing topoi by topological groupoids, J. Pure Appl. Algebra 130 (1998) 223-235 MR1633759

[4] M Crainic, R L Fernandes, Integrability of Lie brackets, Ann. of Math. 157 (2003) 575-620 MR1973056

[5] B Fantechi, L Göttsche, L Illusie, S L Kleiman, N Nitsure, A Vistoli, Fundamental algebraic geometry, Math. Surveys and Monographs 123, Amer. Math. Soc. (2005) MR2222646

[6] J Giraud, Cohomologie non abélienne, Grundlehren der Math. Wissenschaften 179, Springer, Berlin (1971) MR0344253

[7] A Henriques, D Gepner, Homotopy theory of orbispaces arXiv:math/0701916

[8] P T Johnstone, The point of pointless topology, Bull. Amer. Math. Soc. 8 (1983) 41-53 MR682820

[9] P T Johnstone, Stone spaces, Cambridge Studies in Adv. Math. 3, Cambridge Univ. Press (1986) MR861951

[10] P T Johnstone, Sketches of an elephant: a topos theory compendium. Vol. 2, Oxford Logic Guides 44, The Clarendon Press Oxford University Press (2002) MR2063092

[11] A Joyal, M Tierney, An extension of the Galois theory of Grothendieck, Mem. Amer. Math. Soc. 51 (1984) vii+71 MR756176

[12] A Kock, I Moerdijk, Presentations of étendues, Cahiers Topologie Géom. Différentielle Catég. 32 (1991) 145-164 MR1142688

[13] J Lurie, Higher topos theory, Annals of Mathematics Studies 170, Princeton Univ. Press (2009) MR2522659

[14] S Mac Lane, I Moerdijk, Sheaves in geometry and logic, Springer, New York (1994) MR1300636

[15] D Metzler, Topological and smooth stacks arXiv:math/0306176

[16] I Moerdijk, The classifying topos of a continuous groupoid. I, Trans. Amer. Math. Soc. 310 (1988) 629-668 MR973173

[17] I Moerdijk, Foliations, groupoids and Grothendieck étendues, Rev. Acad. Cienc. Zaragoza 48 (1993) 5-33 MR1268130 
[18] I Moerdijk, J Mrčun, Introduction to foliations and Lie groupoids, Cambridge Studies in Advanced Mathematics 91, Cambridge Univ. Press (2003) MR2012261

[19] D A Pronk, Etendues and stacks as bicategories of fractions, Compositio Math. 102 (1996) 243-303 MR1401424

[20] H-H Tseng, C Zhu, Integrating Lie algebroids via stacks, Compos. Math. 142 (2006) 251-270 MR2197411

Max Planck Institute for Mathematics

Vivatsgasse 7, D-53113 Bonn, Germany

davidcarchedi@gmail.com

http://people.mpim-bonn.mpg.de/carchedi/

Received: 24 April 2012 Revised: 16 October 2012 